\title{
Design, Synthesis, and Analysis of Minor Groove Binder Pyrrolepolyamide-2' -Deoxyguanosine Hybrids
}

\author{
Etsuko Kawashima, ${ }^{1}$ Yusuke Ohba, ${ }^{1}$ Yusuke Terui, ${ }^{2}$ and Kazuo Kamaike ${ }^{1}$ \\ ${ }^{1}$ School of Pharmacy, Tokyo University of Pharmacy and Life Sciences, 1432-1 Horinouchi, Hachioji, Tokyo 192-0392, Japan \\ ${ }^{2}$ Faculty of Pharmaceutical Sciences, Chiba Institute of Science, 15-8 Shiomi-cho, Choshi, Chiba 288-0025, Japan \\ Correspondence should be addressed to Etsuko Kawashima, kawasima@toyaku.ac.jp
}

Received 22 May 2009; Revised 19 September 2009; Accepted 9 November 2009

Academic Editor: Mitsuo Sekine

Copyright (C) 2010 Etsuko Kawashima et al. This is an open access article distributed under the Creative Commons Attribution License, which permits unrestricted use, distribution, and reproduction in any medium, provided the original work is properly cited.

Pyrrolepolyamide-2' -deoxyguanosine hybrids (Hybrid 2 and Hybrid 3) incorporating the 3-aminopropionyl or 3-aminopropyl linker were designed and synthesized on the basis of previously reported results of a pyrrolepolyamide-adenosine hybrid (Hybrid 1). Evaluation of the DNA binding sequence selectivity of pyrrolepolyamide-2'-deoxyguanosine hybrids was performed by $C D$ spectral and $T_{\mathrm{m}}$ analyses. It was shown that Hybrid 3 possessed greater binding specificity than distamycin A, Hybrid 1 and Hybrid 2.

\section{Introduction}

Organic compounds capable of controlling gene expression may potentially be used as viable gene therapy agents. In this regard, one of the most important requirements the drug must possess is the ability to selectively distinguish target sequences from all other sequences within the genome. The development of antisense or antigene drugs comprising synthetic oligonucleotides has been investigated [1]. Furthermore, it was reported that pyrrolepolyamide molecules such as distamycin A (Dst) and netropsin (Net), which bind to the minor groove of DNA, can interfere with gene expression through sequence-specific recognition of DNA [2-18] (Figure 1). Baraldi et al. reported on the design, synthesis, and biological activity of hybrid compounds comprising a combination of Dst and uramustine (uracil mustard) [14]. Uramustine interacts with GC-rich regions and can alkylate guanine-N7. The hybrid compounds exhibited enhanced antitumor activity against the K562 Human Leukemia Cell Line compared to both distamycin A and uramustine derivatives. In this case, the interaction with DNA tends to be dominated by minor groove binding of the Dst moiety.
Given these findings, we expected that nucleosides bearing a minor groove binder (MGB) such as a pyrrolepolyamide, which possesses high affinity for the AAATT sequence, might be able to regulate gene expression. When the MGB polyamide-nucleoside hybrid acts on dsDNA, it is expected that complex formation would involve high affinity and sequence selectivity through minor groove-binding of the polyamide moiety and interactions between dsDNA and the nucleoside moiety of the hybrid such as those involving Hoogsteen-type hydrogen bonding. On the other hand, if assuming the hybrid is incorporated into DNA during DNA biosynthesis, it is expected that DNA replication and transcription would be obstructed through minor groovebinding of the hybrid polyamide moiety, incorporated near the target sequence of the DNA. Therefore, it is preferable that the pyrrolepolyamide be combined with the nucleoside site located on the minor groove side of a DNA duplex, for example, the $2^{\prime}$-position of the sugar moiety and the 2 -exocyclic amino group of the guanine base. With this in mind, we previously designed and synthesized Hybrid 1 , where the pyrrolepolyamide is linked at the $2^{\prime}$ hydroxyl group of adenosine, as a lead compound for potential use as 
<smiles>Cn1cc(NC(=O)c2cc(NC(=O)CNC(N)[NH3+])cn2C)cc1C(=O)NCCC(N)=[18O]</smiles><smiles></smiles><smiles>CC1CC2OC(CO)C(CC2n2cnc3c(=O)[nH]c(NC(=O)CCNC(=O)c4cc(NC(=O)c5cc(NC(=O)c6cc(NC=O)cn6C)cn5C)cn4C)nc32)O1</smiles><smiles>Cn1ccc(NC(=O)c2cc(NC(=O)c3cc(NC(=O)c4cc(NC(=O)NCCCNc5nc6c(ncn6C6COC(CO)C(O)C6)c(=O)[nH]5)n(C)c4)cn3C)cn2C)c1</smiles>

Figure 1: Structures of Distamycin A, Netropsin, Hybrid 1, Hybrid 2, and Hybrid 3.

a new gene therapy agent $[19,20]$. Evaluation of the DNAbinding activity of Hybrid $\mathbf{1}$ by circular dichroism (CD) spectral and $T_{\mathrm{m}}$ value analyses showed that although the $\Delta T_{m}$ value $\left(\mathrm{ca} .2^{\circ} \mathrm{C}\right.$, Table 1$)$ was small, Hybrid 1 displayed greater binding specificity than Dst and Net.

On the basis of these results, and for the purpose of developing a gene control agent through interaction with the DNA duplex and/or incorporation into DNA during DNA biosynthesis, we designed MGB polyamide2'-deoxynucleoside hybrids, such as Hybrid 2 and Hybrid
3 combined with a pyrrolepolyamide using a linker at the 2-exocyclic amino group of the guanine base (Figure 1). When considering a DNA strand with incorporated hybrid during DNA biosynthesis, it is expected that a short linker might result in high stabilization of the dsDNA. Therefore, we designed Hybrid 2 containing a 3-aminopropionyl linker used to the head-to-tail hairpin polyamide reported by Mrksich et al. [21]. Moreover, for investigations concerning the stabilization of dsDNA with the complementary strand, we designed Hybrid 3 containing an alkyl linker connected 
directly at the 2-exocyclic amino group of the guanine base. Here we report on the synthesis and interaction of these hybrids on DNA duplexes.

\section{Materials and Methods}

2.1. General. Column chromatography was performed on silica gel (Silica gel N60, purchased from Kanto Chemical Co., Inc.) using methanol/chloroform as solvent systems. Melting points were determined using a Yanaco Micromelting-point apparatus and are uncorrected. ${ }^{1} \mathrm{H}-\mathrm{N} . \mathrm{m} . \mathrm{r}$. and ${ }^{31}$ P-n.m.r. spectra were recorded on a Bruker DRX 400 apparatus. ${ }^{19}$ F-N.m.r. spectra were recorded on a Varian MERCURY 300 apparatus. Mass spectra were recorded on a Micromass Q-Tof Ultima API apparatus. Elemental analyses were achieved using an Elemental Vavio EL apparatus. Circular dichroism (CD) spectra were recorded on a JASCO J-720 spectropolarimeter. UV melting curves were measured using a Shimazu TMSPC-8/UV-1600 apparatus. DNA oligonucleotides were purchased from Hokkaido System Science Co., Ltd. Methyl 4-nitropyrrole-2-carboxylate (5) and 4-[(tertbutoxycarbonyl)amino]-1-methylpyrrole-2-carboxylic acid (6) were prepared according to the procedure described by Baird and Dervan [3]. 3', 5'-O-(Tetraisopropyldisiloxane1,3-diyl)-2'-deoxyguanosine (12) and $3^{\prime}, 5^{\prime}$-di-O-acetyl2-fluoro- $\mathrm{O}^{6}$-[2-(4-nitrophenyl)ethyl]-2'-deoxyinosine (20) were prepared from $2^{\prime}$-deoxyguanosine, as previously described [22-26].

2.2. Methyl 4-[[4-(Tert-butoxycarbonyl)amino-1-methylpyrrol-2-yl]carbonyl]amino-1-methylpyrrole-2-carboxylate (7). Compound 5 (3.000 g, $16.3 \mathrm{mmol})$ was dissolved in ethyl acetate $(100 \mathrm{~mL})$ and $10 \% \mathrm{Pd} / \mathrm{C}$ catalyst $(1.000 \mathrm{~g})$ was added to the solution. The mixture was stirred under a slight positive pressure of hydrogen for 12 hours at room temperature. The catalyst was removed by filtration through Celite and the filtrate was concentrated in vacuo to give methyl 4-amino-1-methylpyrrole-2-carboxylate, which was subsequently used without purification. Methyl 4-amino-1methylpyrrole-2-carboxylate was dissolved in DMF $(100 \mathrm{~mL})$ and 4-[(tert-butoxycarbonyl)amino]-1-methylpyrrole-2-carboxylic acid (6) (3.920 g, $16.3 \mathrm{mmol})$, EDCI (6.250 g, $32.6 \mathrm{mmol}$ ) and DMAP $(3.980 \mathrm{~g}, 32.6 \mathrm{mmol})$ were added to the solution. After stirring for 3 hours, the solution was diluted with ethyl acetate $(200 \mathrm{~mL})$ and methanol $(20 \mathrm{~mL})$ and washed with $3 \mathrm{M} \mathrm{HCl}$ aq. $(100 \mathrm{~mL} \times 3), \mathrm{H}_{2} \mathrm{O}(100 \mathrm{~mL})$, $5 \% \mathrm{NaHCO}_{3}$ aq. $(100 \mathrm{~mL} \times 2)$ and $\mathrm{H}_{2} \mathrm{O}(100 \mathrm{~mL})$. The organic layer was dried over anhydrous magnesium sulfate and evaporated to dryness. Compound 7 was obtained in $99 \%$ yield $(6.070 \mathrm{~g})$ as a yellow glass; ${ }^{1} \mathrm{H}$-n.m.r. (DMSO- $\left.\mathrm{d}_{6}\right)$ $\delta 9.85$ (s, 1H, -NHCO-), 9.09 (s, 1H, Boc-NH), 7.45 (d, 1H, $J=1.9 \mathrm{~Hz}, \mathrm{Py}-\mathrm{H}), 6.90(\mathrm{~d}, 1 \mathrm{H}, J=1.9 \mathrm{~Hz}, \mathrm{Py}-\mathrm{H}), 6.89$ (s, 1H, Py-H), 6.84 (s, 1H, Py-H), 3.83 (s, 3H, Py-CH $\mathrm{CH}_{3}$, $3.81\left(\mathrm{~s}, 3 \mathrm{H}, \mathrm{Py}_{-} \mathrm{CH}_{3}\right), 3.73\left(\mathrm{~s}, 3 \mathrm{H},-\mathrm{OCH}_{3}\right), 1.45(\mathrm{~s}, 9 \mathrm{H}$, $-\mathrm{CH}_{3}$ of the Boc group); ${ }^{13}$ C-n.m.r. (DMSO-d $\left.\mathrm{d}_{6}\right) \delta 160.80$, $158.42,152.85,122.98,122.60,122.42,120.72,118.49$, $117.15,108.37,103.82,78.30,50.90,36.13,36.01,28.19$; ESI-TOF MS calcd for $\mathrm{C}_{18} \mathrm{H}_{25} \mathrm{~N}_{4} \mathrm{O}_{5}(\mathrm{M}+\mathrm{H})^{+}$377.1825, found 377.1826; Anal. Calcd for $\mathrm{C}_{18} \mathrm{H}_{24} \mathrm{~N}_{4} \mathrm{O}_{5}+0.5 \mathrm{H}_{2} \mathrm{O}$ : C, 56.09; H, 6.54; N, 14.54, found C, 56.18; H, 6.28; N, 14.51 .

2.3. Methyl 4-[[4-[[4-(Tert-butoxycarbonyl)amino-1-methylpyrrol-2-yl]carbonyl]amino -1- methylpyrrol - 2 - yl] carbonyl] amino-1-methylpyrrole-2-carboxylate (8). Compound 7 $(3.000 \mathrm{~g}, 16.3 \mathrm{mmol})$ was dissolved in ethyl acetate $(20 \mathrm{~mL}) / \mathrm{methanol}(4 \mathrm{~mL})$ and acetyl chloride $(5.7 \mathrm{~mL}$, $80 \mathrm{mmol}$ ) was added to the solution at $0^{\circ} \mathrm{C}$. The mixture was stirred for 30 minutes. The reaction solution was concentrated in vacuo to give methyl 4-[(4-amino-1-methylpyrrol-2-yl) carbonyl] amino-1-methylpyrrole- 2 -carboxylate, which was subsequently used without purification. Methyl 4-[(4-amino-1-methylpyrrol-2-yl)carbonyl]amino1-methylpyrrole-2-carboxylate was dissolved in DMF $(50 \mathrm{~mL})$ and 4-[(tert-butoxycarbonyl)amino]-1-methylpyrrole-2-carboxylic acid (6) (2.110 g, $8.77 \mathrm{mmol})$, EDCI $(3.060 \mathrm{~g}, 15.9 \mathrm{mmol})$ and DMAP $(2.920 \mathrm{~g}, 23.9 \mathrm{mmol})$ were added to the solution. After stirring for 3 hours, the solution was diluted with ethyl acetate $(200 \mathrm{~mL}) /$ methanol $(20 \mathrm{~mL})$ and washed with $3 \mathrm{M} \mathrm{HCl}$ aq. $(100 \mathrm{~mL} \times 3), \mathrm{H}_{2} \mathrm{O}(100 \mathrm{~mL})$, $5 \% \mathrm{NaHCO}_{3}$ aq. $(100 \mathrm{~mL} \times 2)$ and $\mathrm{H}_{2} \mathrm{O}(100 \mathrm{~mL})$. The organic layer was dried over anhydrous magnesium sulfate and evaporated to dryness. Compound $\mathbf{8}$ was obtained in $98 \%$ yield $(3.900 \mathrm{~g}) ;{ }^{1} \mathrm{H}-n . m . r .\left(\mathrm{DMSO}_{-} \mathrm{d}_{6}\right) \delta 9.92(\mathrm{~s}, 1 \mathrm{H}$, -NHCO-), 9.87 (s, 1H, -NHCO-), 9.08 (s, 1H, Boc-NH), 7.47 (d, 1H, J = 1.9 Hz, Py-H), 7.22 (d, 1H, J = 1.6 Hz, Py$\mathrm{H}), 7.08(\mathrm{~d}, 1 \mathrm{H}, J=1.6 \mathrm{~Hz}, \mathrm{Py}-\mathrm{H}), 6.92(\mathrm{~d}, 1 \mathrm{H}, J=1.9 \mathrm{~Hz}$, Py-H), 6.90 (s, 1H, Рy-H), 6.85 (s, 1H, Py-H), 3.85 (s, $3 \mathrm{H}, \mathrm{Py}-\mathrm{CH}_{3}$ ), 3.84 (s, 3H, Py- $\mathrm{CH}_{3}$ ), 3.82 (s, 3H, $\mathrm{Py}-\mathrm{CH}_{3}$ ), $3.74\left(\mathrm{~s}, 3 \mathrm{H},-\mathrm{OCH}_{3}\right), 1.46\left(\mathrm{~s}, 9 \mathrm{H},-\mathrm{CH}_{3}\right.$ of the Boc group); ${ }^{13}$ C-n.m.r. (DMSO-d 6 ) $\delta$ 160.81, 158.52, 158.44, 152.88, $123.01,122.83,122.52,122.34,120.74,118.52,117.08$, $108.38,104.83,103.85,78.29,50.91,36.15,36.04,28.20$.

2.4. 4-[[4-[[4-(Tert-butoxycarbonyl)amino-1-methylpyrrol-2yl] carbonyl ] amino - 1 - methylpyrrol - 2 - yl] carbonyl] amino 1-methylpyrrole-2-carboxylic acid (9). Compound 8 (0.249 g, $0.5 \mathrm{mmol})$ was dissolved in methanol $(2.5 \mathrm{~mL})$ and $2 \mathrm{M}$ $\mathrm{NaOH}$ aq. $(2.5 \mathrm{~mL})$ was added to the solution. The mixture was stirred at $60^{\circ} \mathrm{C}$ for 1 hour. The reaction solution was concentrated in vacuo and diluted with $\mathrm{H}_{2} \mathrm{O}(25 \mathrm{~mL})$, washed with diethyl ether $(10 \mathrm{~mL} \times 3)$, and neutralized with $10 \% \mathrm{HCl}$ aq. to $\mathrm{ca}$. $\mathrm{pH} 2$. Compound 9 was extracted with ethyl acetate $(20 \mathrm{~mL} \times 3)$ from the resulting solution. The organic layer was washed with $\mathrm{H}_{2} \mathrm{O}(30 \mathrm{~mL})$, dried over anhydrous magnesium sulfate and evaporated to dryness. Compound 9 was obtained in $95 \%$ yield $(0.230 \mathrm{~g})$ as a white powder; m.p. $175-178^{\circ} \mathrm{C}$; ${ }^{1} \mathrm{H}$-n.m.r. $\left(\mathrm{DMSO}_{-} \mathrm{d}_{6}\right) \delta 12.13(\mathrm{~s}$, $1 \mathrm{H}, \mathrm{COOH}), 9.88$ (s, 1H, -NHCO-), 9.85 (s, 1H, -NHCO-), 9.08 (s, 1H, Boc-NH), 7.42 (d, 1H, J = 1.9 Hz, Py-H), 7.21 $(\mathrm{d}, 1 \mathrm{H}, J=1.6 \mathrm{~Hz}, \mathrm{Py}-\mathrm{H}), 7.07$ (d, $1 \mathrm{H}, J=1.6 \mathrm{~Hz}, \mathrm{Py}-\mathrm{H})$, 6.89 (s, 1H, Py-H), 6.85 (d, 1H, J = 1.9 Hz, Py-H), 6.84 (s, $1 \mathrm{H}, \mathrm{Py}-\mathrm{H}$ ), 3.84 (s, 3H, Py- $\mathrm{CH}_{3}$ ), 3.83 (s, 3H, $\left.\mathrm{Py}-\mathrm{CH}_{3}\right), 3.81$ $\left(\mathrm{s}, 3 \mathrm{H}, \mathrm{Py}-\mathrm{CH}_{3}\right), 1.46$ (s, 9H, $-\mathrm{CH}_{3}$ of the Boc group); ${ }^{13} \mathrm{C}-$ n.m.r. $\left(\right.$ DMSO-d $\left._{6}\right) \delta 161.95,158.45,158.42,152.86,122.81$, 
$122.70,122.57,122.32,122.28,120.25,119.50,118.44$, $117.06,108.39$, 104.77, 103.82, 48.59, 36.11, 36.02, 28.20; ESI-TOF MS calcd for $\mathrm{C}_{23} \mathrm{H}_{29} \mathrm{~N}_{6} \mathrm{O}_{6}(\mathrm{M}+\mathrm{H})^{+}$485.2149, found 485.2152; Anal. Calcd for $\mathrm{C}_{23} \mathrm{H}_{28} \mathrm{~N}_{6} \mathrm{O}_{6}+\mathrm{H}_{2} \mathrm{O}$ : C, 54.97; H, 6.02; N, 16.72, found C, 55.18; H, 6.22; N, 16.06 .

\subsection{4-[[4-[(4-Formylamino-1- methylpyrrol-2-yl)carbonyl]} amino-1-methylpyrrol-2-yl]carbonyl]amino-1-methylpyrrole2-carboxylic acid (10). Compound 9 (77 mg, $0.19 \mathrm{mmol}$ ) was dissolved in methanol $(3 \mathrm{~mL})$ and acetyl chloride $(0.85 \mathrm{~mL}, 12 \mathrm{mmol})$ was added to the solution at $0^{\circ} \mathrm{C}$. After stirring for 30 minutes, the reaction solution was concentrated in vacuo to dryness. The residue was dissolved in methanol $(1 \mathrm{~mL})$ and $N$-formylimidazole, prepared by the treatment of formic acid $(12.9 \mu \mathrm{L}, 0.34 \mathrm{mmol})$ with $1,1^{\prime}$-carbonyldiimidazole (CDI) $(36 \mathrm{mg}, 0.22 \mathrm{mmol})$ in THF $(1.5 \mathrm{~mL})$ at room temperature for 5 minutes [11], was added to the solution at $-40^{\circ} \mathrm{C}$. After stirring at $-40^{\circ} \mathrm{C}$ for 30 minutes, the solution was diluted with $5 \% \mathrm{NaHCO}_{3}$ aq. $(10 \mathrm{~mL})$, washed with ethyl acetate $(10 \mathrm{~mL} \times 2)$, and neutralized with $10 \% \mathrm{HCl}$ aq. to $c a . \mathrm{pH} \mathrm{2}$. Compound 10 was extracted with ethyl acetate $(20 \mathrm{~mL} \times 3)$ from the resulting solution. The organic layer was washed with $\mathrm{H}_{2} \mathrm{O}$ $(30 \mathrm{~mL})$, dried over anhydrous magnesium sulfate, and evaporated to dryness. The residue was subjected to silica gel column chromatography using a methanol/chloroform (1: 9, v/v) solvent system. Compound $\mathbf{1 0}$ was obtained in $57 \%$ yield $\left(45 \mathrm{mg}\right.$ ) as a white powder; m.p. $178-181^{\circ} \mathrm{C}$; ${ }^{1}$ H-n.m.r. (DMSO-d 6 ) $\delta 12.17$ (br, 1H, -COOH), 10.05 (d, $1 \mathrm{H}, J=1.6 \mathrm{~Hz},-\mathrm{NHCHO}), 9.92$ (s, 1H, -NHCO-), 9.89 (s, $1 \mathrm{H},-\mathrm{NHCO}-), 8.13$ (s, $1 \mathrm{H}, J=1.6 \mathrm{~Hz},-\mathrm{CHO}), 7.42(\mathrm{~d}, 1 \mathrm{H}$, $J=1.7 \mathrm{~Hz}, \mathrm{Py}-\mathrm{H}), 7.23(\mathrm{~d}, 1 \mathrm{H}, J=1.7 \mathrm{~Hz}, \mathrm{Py}-\mathrm{H}), 7.20(\mathrm{~d}$, $1 \mathrm{H}, J=1.7 \mathrm{~Hz}, \mathrm{Py}-\mathrm{H}), 7.06(\mathrm{~d}, 1 \mathrm{H}, J=1.7 \mathrm{~Hz}, \mathrm{Py}-\mathrm{H}), 6.92$ $(\mathrm{d}, 1 \mathrm{H}, J=1.7 \mathrm{~Hz}, \mathrm{Py}-\mathrm{H}), 6.85(\mathrm{~d}, 1 \mathrm{H}, J=1.7 \mathrm{~Hz}, \mathrm{Py}-\mathrm{H})$, 3.85 (s, 3H, Py-CH $\left.\mathrm{CH}_{3}\right), 3.84$ (s, 3H, $\left.\mathrm{Py}-\mathrm{CH}_{3}\right), 3.38(\mathrm{~s}, 3 \mathrm{H}$, Py-CH ${ }_{3}$ ); ${ }^{13}$ C-n.m.r. (DMSO-d 6 ) $\delta 162.70,162.13,158.45$, $158.31,157.91,122.98,122.67,122.65,122.17,120.76$, $120.16,119.75,118.51,118.44,108.34,104.74,103.98$, 36.13, 36.06; ESI-TOF MS calcd for $\mathrm{C}_{19} \mathrm{H}_{21} \mathrm{~N}_{6} \mathrm{O}_{5}(\mathrm{M}+\mathrm{H})^{+}$ 413.1573, found 413.1579 .

2.6. 3-[[4-[[4-[(4-Formylamino-1-methylpyrrol-2-yl)carbonyl] amino-1-methylpyrrol-2-yl]carbonyl]amino-1-methylpyrrol-2-yl]carboxyl]aminopropionic acid (11). Compound 9 $(0.100 \mathrm{~g}, 0.206 \mathrm{mmol})$ was dissolved in DMF $(2 \mathrm{~mL})$ and ethyl $\beta$-alanine hydrochloride $(35 \mathrm{mg}, 0.227 \mathrm{mmol})$, EDCI (47 mg, $0.247 \mathrm{mmol}$ ), and DMAP (60 mg, $0.494 \mathrm{mmol})$ were added to the solution. After stirring for 3 hours, the solution was diluted with ethyl acetate $(20 \mathrm{~mL})$ and washed with $10 \% \mathrm{HCl}$ aq. $(10 \mathrm{~mL} \times 3), \mathrm{H}_{2} \mathrm{O}(10 \mathrm{~mL})$, $5 \% \mathrm{NaHCO}_{3}$ aq. $(10 \mathrm{~mL} \times 3)$, and $\mathrm{H}_{2} \mathrm{O}(10 \mathrm{~mL})$. The organic layer was dried over anhydrous magnesium sulfate and evaporated to dryness. Ethyl 3-[4-[[4-[[4(tert-butoxycarbonyl)amino-1-methylpyrrol-2-yl]carbonyl] amino-1-methylpyrrol-2-yl] carbonyl] amino-1 -methylpyrrol-2-yl]carboxyl]aminopropionate was obtained in $85 \%$ yield (102 mg); ${ }^{1} \mathrm{H}-$ n.m.r. $\left(\mathrm{DMSO}_{6} \mathrm{~d}_{6}\right) \quad \delta 9.87 \quad(\mathrm{~s}, 1 \mathrm{H}$, -NHCO-), 9.85 (s, 1H, -NHCO-), 9.07 (s, 1H, Boc-NH), $8.03(\mathrm{t}, 1 \mathrm{H}, J=6 \mathrm{~Hz}, \mathrm{Py}-\mathrm{NH}), 7.21(\mathrm{~d}, 1 \mathrm{H}, J=1.3 \mathrm{~Hz}, \mathrm{Py}-$ $\mathrm{H}), 7.18(\mathrm{~d}, 1 \mathrm{H}, J=1.5 \mathrm{~Hz}, \mathrm{Py}-\mathrm{H}), 7.03(\mathrm{~d}, 1 \mathrm{H}, J=1.3 \mathrm{~Hz}$, Py-H), 6.89 (s, 1H, Py-H), 6.85 (d, 1H, J = 1.5 Hz, Py-H), 6.84 (s, 1H, Py-H), 4.07 (q, 2H, J = 7.1 Hz, $\mathrm{CH}_{3} \mathrm{CH}_{2}-$ ), 3.84 (s, 3H, Py- $\mathrm{CH}_{3}$ ), 3.81 (s, 3H, Py- $\left.\mathrm{CH}_{3}\right), 3.80$ (s, 3H, $\mathrm{Py}-\mathrm{CH}_{3}$ ), $3.40\left(\mathrm{dt}, 2 \mathrm{H}, J=7 \mathrm{~Hz}\right.$ and $\left.J=6 \mathrm{~Hz},-\mathrm{CH}_{2} \mathrm{NH}-\right), 2.53(\mathrm{t}, 2 \mathrm{H}$, $\left.J=7 \mathrm{~Hz},-\mathrm{COCH}_{2}-\right), 1.46\left(\mathrm{~s}, 9 \mathrm{H},-\mathrm{CH}_{3}\right.$ of the Boc group), $1.19\left(\mathrm{t}, 3 \mathrm{H}, \mathrm{J}=7.1 \mathrm{~Hz}, \mathrm{CH}_{3} \mathrm{CH}_{2-}\right) ;{ }^{13} \mathrm{C}-$ n.m.r. $\left(\mathrm{DMSO}-\mathrm{d}_{6}\right.$ ) $\delta 171.34,161.27,158.45,158.40,152.84,122.82,122.71$, $122.65,122.28,122.16,118.35,117.90,117.00,104.27$, $103.81,59.84,36.00,35.89,34.78,34.02,28.19,14.06$; ESI-TOF MS calcd for $\mathrm{C}_{28} \mathrm{H}_{38} \mathrm{~N}_{7} \mathrm{O}_{7}(\mathrm{M}+\mathrm{H})^{+}$584.2833, found 584.2822 .

Ethyl 3-[4-[[4-[[4-(tert-butoxycarbonyl)amino-1-methylpyrrol-2-yl] carbonyl]amino-1-methylpyrrol -2-yl] carbonyl] amino -1- methylpyrrol -2 - yl] carboxyl] aminopropionate (71.8 $\mathrm{mg}, 0.123 \mathrm{mmol})$ was dissolved in methanol $(1 \mathrm{~mL})$, $2 \mathrm{M} \mathrm{NaOH}$ aq. ( $1 \mathrm{~mL}$ ) was added and the solution was stirred at $60^{\circ} \mathrm{C}$ for 3 hours. The reaction solution was concentrated in vacuo and diluted with $\mathrm{H}_{2} \mathrm{O}(10 \mathrm{~mL})$, washed with diethyl ether $(5 \mathrm{~mL} \times 3)$, and neutralized with $10 \% \mathrm{HCl}$ aq. to $\mathrm{ca}$. $\mathrm{pH}$ 2. The product was extracted with ethyl acetate $(10 \mathrm{~mL} \times 3)$ from the resulting solution. The organic layer was washed with $\mathrm{H}_{2} \mathrm{O}(15 \mathrm{~mL})$, dried over anhydrous magnesium sulfate, and evaporated to dryness. 3-[4-[[4 -[[4 -(tert-Butoxycarbonyl)amino-1 - methylpyrrol2-yl] carbonyl] amino-1-methylpyrrol-2-yl] carbonyl] amino1-methylpyrrol-2-yl] carboxyl] aminopropionic acid was obtained in $74 \%$ yield $(50.2 \mathrm{mg}) ;{ }^{1} \mathrm{H}-$ n.m.r. $\left(\mathrm{DMSO}^{\left.-\mathrm{d}_{6}\right)} \delta\right.$ 11.90 (br, 1H, -COOH), 9.87 (s, 1H, -NHCO-), 9.85 (s, 1H, -NHCO-), 9.07 (s, 1H, Boc-NH), $8.02(\mathrm{t}, 1 \mathrm{H}, J=6 \mathrm{~Hz}$, Py-NH), $7.22(\mathrm{~d}, 1 \mathrm{H}, J=1.6 \mathrm{~Hz}, \mathrm{Py}-\mathrm{H}), 7.18(\mathrm{~d}, 1 \mathrm{H}$, $J=1.7 \mathrm{~Hz}, \mathrm{Py}-\mathrm{H}), 7.03(\mathrm{~d}, 1 \mathrm{H}, J=1.6 \mathrm{~Hz}, \mathrm{Py}-\mathrm{H}), 6.89$ (s, 1H, Py-H), 6.85 (d, 1H, J = 1.7 Hz, Py-H), $6.84(\mathrm{~s}, 1 \mathrm{H}$, Py-H), 3.84 (s, 3H, Py- $\mathrm{CH}_{3}$ ), 3.81 (s, 3H, Py- $\mathrm{CH}_{3}$ ), 3.80 $\left(\mathrm{s}, 3 \mathrm{H}, \mathrm{Py}-\mathrm{CH}_{3}\right), 3.37(\mathrm{dt}, 2 \mathrm{H}, J=7 \mathrm{~Hz}$ and $J=6 \mathrm{~Hz}$, $-\mathrm{CH}_{2} \mathrm{NH}-$ ), 2.45 (t, 2H, J $\left.=7 \mathrm{~Hz},-\mathrm{COCH}_{2}-\right), 1.46(\mathrm{~s}, 9 \mathrm{H}$, $-\mathrm{CH}_{3}$ of the Boc group); ${ }^{13} \mathrm{C}$-n.m.r. (DMSO-d 6 ) $\delta 173.05$, $162.31,158.45,153.71,122.84,122.71,122.68,122.28$, $122.15,118.24,117.85,116.97,104.67,104.28,103.94,78.24$, 59.74, 36.00, 35.91, 34.89, 34.17, 28.19; ESI-TOF MS calcd for $\mathrm{C}_{26} \mathrm{H}_{34} \mathrm{~N}_{7} \mathrm{O}_{7}(\mathrm{M}+\mathrm{H})^{+}$556.2520, found 556.2493.

3-[4-[[4-[ [4-(tert-Butoxycarbonyl)amino-1-methylpyrrol-2-yl] carbonyl] amino-1-methylpyrrol-2-yl] carbonyl]amino-1-methylpyrrol-2-yl] carboxyl] aminopropionic acid (40.6 $\mathrm{mg}, 0.073 \mathrm{mmol})$ was dissolved in methanol $(3 \mathrm{~mL})$ and acetyl chloride $(0.85 \mathrm{~mL}, 80 \mathrm{mmol})$ was added dropwise at $0^{\circ} \mathrm{C}$. After stirring for 30 minutes, the solution was concentrated in vacuo to dryness. The residue was dissolved in methanol $(0.5 \mathrm{~mL})$ and to this was added $\mathrm{N}$-formylimidazole, prepared by the treatment of formic acid $(3.3 \mu \mathrm{L}, 0.088 \mathrm{mmol})$ with $1,1^{\prime}$-carbonyldiimidazole ( $14.2 \mathrm{mg}, 0.088 \mathrm{mmol})$ in $\mathrm{THF}(1 \mathrm{~mL})$ at room temperature for 30 minutes [11], at $-40^{\circ} \mathrm{C}$. After stirring at $-40^{\circ} \mathrm{C}$ for 1 hour, the solution was diluted with ethyl acetate $(10 \mathrm{~mL})$, and washed with sat. $\mathrm{NH}_{4} \mathrm{Cl}$ aq. $(5 \mathrm{~mL} \times 3)$ and $\mathrm{H}_{2} \mathrm{O}(5 \mathrm{~mL})$. The organic layer was dried over anhydrous magnesium sulfate and evaporated to dryness. The residue was subjected to silica gel column chromatography using a 
methanol/chloroform $(1: 9, \mathrm{v} / \mathrm{v})$ solvent system. Compound 11 was obtained in $85 \%$ yield $(30.2 \mathrm{mg}) ;{ }^{1} \mathrm{H}$-n.m.r. (DMSO$\left.\mathrm{d}_{6}\right) \delta 12.00(\mathrm{br}, 1 \mathrm{H},-\mathrm{COOH}), 10.03(\mathrm{~d}, 1 \mathrm{H}, J=1.7 \mathrm{~Hz}$, -NHCHO), 9.91 (s, 1H, -NHCO-), 9.88 (s, 1H, -NHCO-), $8.13(\mathrm{~d}, 1 \mathrm{H}, J=1.7 \mathrm{~Hz},-\mathrm{NHCHO}), 8.00(\mathrm{t}, 1 \mathrm{H}, J=6 \mathrm{~Hz}$, Py-NH), $7.23(\mathrm{~d}, 1 \mathrm{H}, J=1.7 \mathrm{~Hz}, \mathrm{Py}-\mathrm{H}), 7.19(\mathrm{~d}, 1 \mathrm{H}$, $J=1.7 \mathrm{~Hz}, \mathrm{Py}-\mathrm{H}), 7.18(\mathrm{~d}, 1 \mathrm{H}, J=1.7 \mathrm{~Hz}, \mathrm{Py}-\mathrm{H}), 7.03(\mathrm{~d}$, $1 \mathrm{H}, J=1.8 \mathrm{~Hz}, \mathrm{Py}-\mathrm{H}), 6.91(\mathrm{~d}, 1 \mathrm{H}, J=1.8 \mathrm{~Hz}, \mathrm{Py}-\mathrm{H}), 6.85$ $(\mathrm{d}, 1 \mathrm{H}, J=1.7 \mathrm{~Hz}, \mathrm{Py}-\mathrm{H}), 3.84\left(\mathrm{~s}, 3 \mathrm{H}, \mathrm{Py}-\mathrm{CH}_{3}\right), 3.83(\mathrm{~s}$, $\left.3 \mathrm{H}, \mathrm{Py}-\mathrm{CH}_{3}\right), 3.80$ (s, $\left.3 \mathrm{H}, \mathrm{Py}-\mathrm{CH}_{3}\right), 3.37(\mathrm{dt}, 2 \mathrm{H}, \mathrm{J}=7 \mathrm{~Hz}$ and $\left.J=6 \mathrm{~Hz},-\mathrm{CH}_{2} \mathrm{NH}-\right), 2.47\left(\mathrm{t}, 2 \mathrm{H}, J=7 \mathrm{~Hz},-\mathrm{COCH}_{2}-\right)$; ${ }^{13}$ C-n.m.r. (DMSO-d $\left.{ }_{6}\right) \delta 173.00,161.24,158.42,158.27,157$, $85,122.95,122.75,122.13,122.04,120.71,118.39,117.87$, 104.63, 104.24, 103.93, 36.09, 36.05, 35.91, 34.84, 34.07; ESI-TOF MS calcd for $\mathrm{C}_{22} \mathrm{H}_{26} \mathrm{~N}_{7} \mathrm{O}_{6}(\mathrm{M}+\mathrm{H})^{+}$484.1945, found 484.1950 .

2.7. $N^{2}-[3-[[4-[[4-[(4-F o r m y l a m i n o-1-m e t h y l p y r r o l-2-y l) c a-$ rbonyl]amino-1-methylpyrrol-2-yl]carbonyl]amino-1-methylpyrrol-2-yl]carboxyl]aminopropionyl]-3',5'-O-(tetraisopropyldisiloxane-1,3-diyl)-2'-deoxyguanosine (14). Method (1) Compound 11 was treated with $3^{\prime}, 5^{\prime}$-O-(tetraisopropyldisiloxane-1,3-diyl)-2' -deoxyguanosine (12) in the presence of EDCI and DMAP or DCC and HOBT as described for the syntheses of 7 and 18. The coupling reaction did not progress and the desired product 14 was not obtained.

Method (2) $N$-(9-Fluorenylmethoxycarbonyl)- $\beta$-alanine ( $4.580 \mathrm{~g}, 14.7 \mathrm{mmol})$ was dissolved in DMF $(0.25 \mathrm{~mL}) / \mathrm{THF}$ $(25 \mathrm{~mL})$ and oxalyl chloride $(1.8 \mathrm{~mL}, 20 \mathrm{mmol})$ was added to the solution at $0^{\circ} \mathrm{C}$. After stirring for 30 minutes at room temperature, the solution was concentrated in vacuo to give 3-[(9-fluorenylmethoxycarbonyl)amino]propionyl chloride. The residue was dissolved in THF $(10 \mathrm{~mL})$ and added to a solution of $3^{\prime}, 5^{\prime}$-O-(tetraisopropyldisiloxane1,3-diyl)-2'-deoxyguanosine (12) in pyridine $(80 \mathrm{~mL})$. After stirring for 72 hours at room temperature, $\mathrm{H}_{2} \mathrm{O}$ $(10 \mathrm{~mL})$ was added to the solution. The solution was diluted with ethyl acetate $(300 \mathrm{~mL})$ and washed with $\mathrm{H}_{2} \mathrm{O}$ $(100 \mathrm{~mL} \times 3), 5 \% \quad \mathrm{NaHCO}_{3}$ aq. $(100 \mathrm{~mL} \times 3)$, and $\mathrm{H}_{2} \mathrm{O}$ $(100 \mathrm{~mL})$. The organic layer was dried over anhydrous magnesium sulfate and evaporated to dryness. The residue was recrystallized from ethyl acetate. The resulting white crystals were collected by vacuum filtration to give $N^{2}$ - [(9fluorenylmethoxycarbonyl)amino] propionyl-3', $5^{\prime}$-O-(tetraisopropyldisiloxane-1,3-diyl)-2'-deoxyguanosine (13) in $43 \%$ yield $(3.420 \mathrm{~g})$; m.p. $199-200{ }^{\circ} \mathrm{C} ;{ }^{1} \mathrm{H}$-n.m.r. (DMSO-d 6 ) $\delta 12.02$ (br, $\left.1 \mathrm{H}, \mathrm{N}^{2}-\mathrm{H}\right), 11.76$ (br, $\left.1 \mathrm{H}, \mathrm{N}^{1}-\mathrm{H}\right), 8.13$ (s, $1 \mathrm{H}$, $\mathrm{H}-8), 7.85$ (d, $2 \mathrm{H}, J=7.5 \mathrm{~Hz}, \mathrm{Ar}-\mathrm{H}$ of the Fmoc group), $7.66(\mathrm{dd}, 2 \mathrm{H}, J=2.6 \mathrm{~Hz}, J=7.3 \mathrm{~Hz}, \mathrm{Ar}-\mathrm{H}$ of the Fmoc group), $7.42\left(\mathrm{t}, 1 \mathrm{H}, J=6 \mathrm{~Hz},-\mathrm{CH}_{2} \mathrm{NH}-\mathrm{Fmoc}\right), 7.37(\mathrm{t}, 1 \mathrm{H}$, $J=7.5 \mathrm{~Hz}$, Ar-H of the Fmoc group), 7.30 (m, 2H, Ar-H of the Fmoc group), $6.12\left(\mathrm{~m}, 1 \mathrm{H}, \mathrm{H}-1^{\prime}\right), 4.66\left(\mathrm{~m}, 1 \mathrm{H}, \mathrm{H}-3^{\prime}\right)$, $4.29\left(\mathrm{~d}, 2 \mathrm{H}, J=6.7 \mathrm{~Hz},=\mathrm{CH}-\mathrm{CH}_{2}-\right.$ of the Fmoc group), $4.21\left(\mathrm{t}, 1 \mathrm{H}, J=6.7 \mathrm{~Hz},=\mathrm{CH}-\mathrm{CH}_{2}-\right.$ of the Fmoc group), $3.82-3.92\left(\mathrm{~m}, 3 \mathrm{H}, \mathrm{H}-4^{\prime}, 5^{\prime}\right.$, and $\left.5^{\prime \prime}\right), 3.32(\mathrm{dt}, 2 \mathrm{H}, J=6 \mathrm{~Hz}$, $\left.J=6,4 \mathrm{~Hz},-\mathrm{CH}_{2} \mathrm{CH}_{2} \mathrm{NH}-\mathrm{Fmoc}\right), 2.64\left(\mathrm{~m}, 1 \mathrm{H}, \mathrm{H}-2^{\prime}\right), 2.54$ $\left(\mathrm{t}, 2 \mathrm{H}, J=6,4 \mathrm{~Hz},-\mathrm{COCH}_{2} \mathrm{CH}_{2}-\right), 2.50\left(\mathrm{~m}, 1 \mathrm{H}, \mathrm{H}-2^{\prime \prime}\right)$, $1.01\left(\mathrm{~m}, 28 \mathrm{H},-\mathrm{CH}\left(\mathrm{CH}_{3}\right)_{2}\right.$ of the TIPDS group); ${ }^{13} \mathrm{C}$-n.m.r. $\left(\mathrm{DMSO}_{\mathrm{d}}\right) \delta 174.39,156.02,154.78,148.06,147.87,143.82$, $140.68,136.68,136.73,127.50,126.96,125.07,120.29$, $120.01,84.64,81.37,70.57,65.36,61.99,46.67,36.50,36.26$, $17.29,17.23,17.02,16.84,16.76,12.66,12.51,12.17,11.94$; ESI-TOF MS calcd for $\mathrm{C}_{40} \mathrm{H}_{55} \mathrm{~N}_{6} \mathrm{O}_{8} \mathrm{Si}_{2}(\mathrm{M}+\mathrm{H})^{+}$803.3620, found 803.3674; Anal. Calcd for $\mathrm{C}_{40} \mathrm{H}_{54} \mathrm{~N}_{6} \mathrm{O}_{8} \mathrm{Si}_{2}+\mathrm{H}_{2} \mathrm{O}$ : C, 58.51; H, 6.87; N, 10.24, found C, 58.67; H, 6.91; N,10.43.

Compounds 10 (214 mg, $0.27 \mathrm{mmol})$ and $13(100 \mathrm{mg}$, $0.24 \mathrm{mmol}$ ) were dissolved in DMF ( $5 \mathrm{~mL}$ ) and EDCI (93 mg, $0.48 \mathrm{mmol}$ ) and DMAP (150 mg, $1.23 \mathrm{mmol}$ ) were added to the solution. After stirring for 24 hours, the solution was diluted with ethyl acetate $(50 \mathrm{~mL}) / \mathrm{methanol}(3 \mathrm{~mL})$ and washed with $\mathrm{H}_{2} \mathrm{O}(30 \mathrm{~mL} \times 3)$. The organic layer was dried over anhydrous magnesium sulfate and evaporated to dryness. The residue was subjected to silica gel column chromatography using a methanol/chloroform (1:9, v/v) solvent system to give 14 (195 mg, 83\% yield); ${ }^{1} \mathrm{H}$-n.m.r. $\left(\right.$ DMSO$\left.-\mathrm{d}_{6}\right) \delta 12.05$ (br, $\left.1 \mathrm{H}, \mathrm{N}^{2}-\mathrm{H}\right), 11.79$ (br, $\left.1 \mathrm{H}, \mathrm{N}^{1}-\mathrm{H}\right)$, 10.04 (s, 1H, -NHCHO), 9.90 (s, 1H, -NHCO-), 9.88 (s, 1H, -NHCO-), 8.13 (s, 1H, H-8), 8.13 (s, 1H, -CHO), 8.11 (t, 1H, $\left.J=5.7 \mathrm{~Hz},-\mathrm{CH}_{2} \mathrm{NHCO}-\right), 7.22(\mathrm{~d}, 1 \mathrm{H}, J=1.6 \mathrm{~Hz}, \mathrm{Py}-\mathrm{H})$, $7.20(\mathrm{~m}, 2 \mathrm{H}, \mathrm{Py}-\mathrm{H} \times 2), 7.03(\mathrm{~d}, 1 \mathrm{H}, J=1.7 \mathrm{~Hz}, \mathrm{Py}-\mathrm{H}), 6.91$ $(\mathrm{d}, 1 \mathrm{H}, J=1.8 \mathrm{~Hz}, \mathrm{Py}-\mathrm{H}), 6.86(\mathrm{~d}, 1 \mathrm{H}, J=1.6 \mathrm{~Hz}, \mathrm{Py}-\mathrm{H})$, $6.15\left(\mathrm{dd}, 1 \mathrm{H}, J=3.5 \mathrm{~Hz}, J=7.1 \mathrm{~Hz}, \mathrm{H}-1^{\prime}\right), 4.66(\mathrm{dt}, 1 \mathrm{H}$, $\left.J=7 \mathrm{~Hz}, J=7.4 \mathrm{~Hz}, \mathrm{H}-3^{\prime}\right), 3.83-3.93\left(\mathrm{~m}, 3 \mathrm{H}, \mathrm{H}-4^{\prime}, 5^{\prime}\right.$, and $\left.5^{\prime \prime}\right), 3.84\left(\mathrm{~s}, 6 \mathrm{H}, \mathrm{Py}-\mathrm{CH}_{3} \times 2\right), 3.79\left(\mathrm{~s}, 3 \mathrm{H}, \mathrm{Py}-\mathrm{CH}_{3}\right), 3.50(\mathrm{dt}$, $\left.2 \mathrm{H}, J=6 \mathrm{~Hz}, J=6.4 \mathrm{~Hz},-\mathrm{CH}_{2} \mathrm{CH}_{2} \mathrm{NH}-\right), 2.75\left(\mathrm{~m}, 2 \mathrm{H}, \mathrm{H}-2^{\prime}\right.$ and $\left.2^{\prime \prime}\right), 2.70\left(\mathrm{t}, 2 \mathrm{H}, \mathrm{J}=6,4 \mathrm{~Hz},-\mathrm{COCH}_{2} \mathrm{CH}_{2}-\right), 1.01(\mathrm{~m}$, $28 \mathrm{H},-\mathrm{CH}\left(\mathrm{CH}_{3}\right)_{2}$ of the TIPDS group); ${ }^{13} \mathrm{C}$-n.m.r. (DMSO$\left.\mathrm{d}_{6}\right) \delta 174.77,161.36,158.43,158.28,157.85,154.83,148.14$, $148.01,136.76,122.96,122.74,122.65,122.19,122.07$, $120.73,120.27,118.41,117.97,104.65,104.34,103.94,84.63$, $81.37,70.55,61.96,36.59,36.10,36.06,35.91,34.63,17.32$, $17.16,17.05,16.87,16.80,12.67,12.52$, 12.18, 11.96; ESITOF MS calcd for $\mathrm{C}_{44} \mathrm{H}_{63} \mathrm{~N}_{12} \mathrm{O}_{10} \mathrm{Si}_{2}(\mathrm{M}+\mathrm{H})^{+}$975.4329, found 975.4271 .

2.8. $N^{2}-[3-[[4-[[4-[(4-F o r m y l a m i n o-1-m e t h y l p y r r o l-2-y l) c a-$ rbonyl]amino-1-methylpyrrol-2-yl]carbonyl] amino-1 - methylpyrrol - 2 - yl] carboxyl] aminopropionyl]-2' -deoxyguanosine (Hybrid 2). Compound 14 (50 mg, $0.051 \mathrm{mmol}$ ) was treated with $0.2 \mathrm{M}$ tetraethylammonium fluoride in THF $(3 \mathrm{~mL})$ and stirred at room temperature for 30 minutes. The solution was concentrated in vacuo to dryness and the residue was subjected to silica gel column chromatography using a methanol/chloroform (1:4, v/v) solvent system to give Hybrid 2 (37.3 mg, quantitative yield); ${ }^{1} \mathrm{H}$-n.m.r. $\left(\mathrm{DMSO}_{\mathrm{d}}\right) \delta 12.04$ (br, $\left.1 \mathrm{H}, \mathrm{N}^{2}-\mathrm{H}\right), 11.74$ (br, $\left.1 \mathrm{H}, \mathrm{N}^{1}-\mathrm{H}\right)$, 10.03 (s, 1H, -NHCHO), 9.90 (s, 1H, -NHCO-), 9.88 (s, 1H, -NHCO-), 8.23 (s, 1H, H-8), 8.13 (s, 1H, -CHO), 8.11 (t, 1H, $\left.J=5.5 \mathrm{~Hz},-\mathrm{CH}_{2} \mathrm{NHCO}-\right), 7.22(\mathrm{~d}, 1 \mathrm{H}, J=1.6 \mathrm{~Hz}, \mathrm{Py}-\mathrm{H})$, $7.20(\mathrm{~m}, 2 \mathrm{H}, \mathrm{Py}-\mathrm{H} \times 2), 7.02(\mathrm{~d}, 1 \mathrm{H}, J=1.7 \mathrm{~Hz}, \mathrm{Py}-\mathrm{H}), 6.91$ $(\mathrm{d}, 1 \mathrm{H}, J=1.7 \mathrm{~Hz}, \mathrm{Py}-\mathrm{H}), 6.85(\mathrm{~d}, 1 \mathrm{H}, J=1.6 \mathrm{~Hz}, \mathrm{Py}-\mathrm{H})$, $6.20\left(\mathrm{t}, 1 \mathrm{H}, J=6.7 \mathrm{~Hz}, \mathrm{H}-1^{\prime}\right), 5.28\left(\mathrm{br}, 1 \mathrm{H}, 5^{\prime}-\mathrm{OH}\right), 4.66$ $\left(\mathrm{t}, 1 \mathrm{H}, J=5.4 \mathrm{~Hz}, \mathrm{H}-3^{\prime}\right), 4.36\left(\mathrm{br}, 1 \mathrm{H}, 3^{\prime}-\mathrm{OH}\right), 3.84(\mathrm{~s}, 6 \mathrm{H}$, $\left.\mathrm{Py}_{-} \mathrm{CH}_{3} \times 2\right), 3.82\left(\mathrm{~m}, 1 \mathrm{H}, \mathrm{H}-4^{\prime}\right), 3.79$ (s, 3H, Py-CH$), 3.54$ 
$\left(\mathrm{m}, 2 \mathrm{H}, \mathrm{H}-5^{\prime}\right.$, and $\left.5^{\prime \prime}\right), 3.50(\mathrm{dt}, 2 \mathrm{H}, J=5.5 \mathrm{~Hz}, J=6.5 \mathrm{~Hz}$, $\left.-\mathrm{CH}_{2} \mathrm{CH}_{2} \mathrm{NH}-\right), 2.71\left(\mathrm{t}, 2 \mathrm{H}, \mathrm{J}=6,5 \mathrm{~Hz},-\mathrm{COCH}_{2} \mathrm{CH}_{2}-\right)$ $2.28\left(\mathrm{~m}, 2 \mathrm{H}, \mathrm{H}-2^{\prime}\right.$ and $\left.2^{\prime \prime}\right) ;{ }^{13} \mathrm{C}-$ n.m.r. $\left(\mathrm{DMSO}-\mathrm{d}_{6}\right) \delta 174.74$, $161.35,158.41,158.27,157.85,154.83,148.35,147.87$, $137.45,122.95,122.73,122.65,122.16,122.04,120.71$, $120.18,118.37,117.96,104.64,104.33,103.94,87.72,82.95$, 70.45, 61.46, 36.58, 36.08, 36.05, 35.89, 34.63; ESI-TOF MS calcd for $\mathrm{C}_{32} \mathrm{H}_{37} \mathrm{~N}_{12} \mathrm{O}_{10}(\mathrm{M}+\mathrm{H})^{+} 733.2806$, found 733.2841; Anal. Calcd for $\mathrm{C}_{32} \mathrm{H}_{36} \mathrm{~N}_{12} \mathrm{O}_{9}+4 \mathrm{H}_{2} \mathrm{O}$ : C, 47.76; $\mathrm{H}, 5.51 ; \mathrm{N}$, 20.89, found C, 47.79; H, 5.84; N, 21.26.

2.9. 9-Fluorenylmethyl 3-aminopropyl-1-carbamate hydrochloride (17). 9-Fluorenylmethyl chloroformate (15) (5.174 g, $20 \mathrm{mmol}$ ) was dissolved in 1,4-dioxane $(50 \mathrm{~mL})$, and sodium phenoxide $3 \mathrm{H}_{2} \mathrm{O}(3.573 \mathrm{~g}, 21 \mathrm{mmol})$ was added to the solution. After stirring for 1.5 hours at room temperature, the solution was concentrated in vacuo and ethyl acetate $(100 \mathrm{~mL})$ was added. The solution was washed with $\mathrm{H}_{2} \mathrm{O}$ $(50 \mathrm{~mL} \times 2)$ and sat. $\mathrm{NaOH}$ aq. $(50 \mathrm{~mL})$. The organic layer was dried over anhydrous magnesium sulfate and evaporated to dryness. The residue was subjected to crystallization from ethyl acetate, collected by vacuum filtration, and dried in vacuo to yield 9-fluorenylmethyl phenyl carbonate (16) (5.825 g, 92\% yield) as a white solid, m.p. $132-133{ }^{\circ} \mathrm{C} ;{ }^{1} \mathrm{H}-$ n.m.r. $\left(\mathrm{DMSO}^{\left.-\mathrm{d}_{6}\right)} \delta 7.92(\mathrm{~d}, 2 \mathrm{H}, J=7.5 \mathrm{~Hz}, \mathrm{Ar}-\mathrm{H}\right.$ of the Fmoc group $\times 2), 7.68(\mathrm{~d}, 2 \mathrm{H}, J=7.5 \mathrm{~Hz}$, Ar-H of the Fmoc group $\times 2), 7.15-7.46(\mathrm{~m}, 9 \mathrm{H}, \mathrm{Ph}-\mathrm{H} \times 5$ and $\mathrm{Ar}-$ $\mathrm{H}$ of the Fmoc group $\times 4), 4.68(\mathrm{~d}, 2 \mathrm{H}, J=6.1 \mathrm{~Hz}$, $=\mathrm{CH}-\mathrm{CH}_{2}-$ of the Fmoc group), $4.39(\mathrm{t}, 1 \mathrm{H}, J=6.1 \mathrm{~Hz}$, $=\mathrm{CH}-\mathrm{CH}_{2}-$ of the Fmoc group); ${ }^{13} \mathrm{C}-$ n.m.r. $\left(\right.$ DMSO- $\left.\mathrm{d}_{6}\right)$ $\delta 152.87,150.61,143.15,140.84,129.57,127.78,127.18$, $126.13,124.94,121.11,120.22,69.38,46.28$; ESI-TOF MS calcd for $\mathrm{C}_{21} \mathrm{H}_{16} \mathrm{O}_{3}(\mathrm{M}+\mathrm{Na})^{+}$339.0997, found 339.0986; Anal. Calcd for $\mathrm{C}_{21} \mathrm{H}_{16} \mathrm{O}_{3}$ : C, 79.73; H, 5.10, found C, 79.79; H, 5.11 .

9-Fluorenylmethyl phenyl carbonate (16) (3.763 g, $12 \mathrm{mmol})$ was suspended in methanol $(50 \mathrm{~mL})$ and propane-1,3-diamine $(1.0 \mathrm{~mL}, 12 \mathrm{mmol})$ was added to the solution. After stirring for 4 hours at room temperature, pyridinium hydrochloride $(3.005 \mathrm{~g}, 26 \mathrm{mmol})$ was added and the solution stirred for 10 minutes. The solution was concentrated in vacuo and the residue was subjected to silica gel column chromatography using a methanol/chloroform (1 : $4, \mathrm{v} / \mathrm{v})$ solvent system to give $17(3.250 \mathrm{~g}, 86 \%$ yield $)$ as a white powder; m.p. $129-130{ }^{\circ} \mathrm{C} ;{ }^{1} \mathrm{H}$-n.m.r. (DMSO- $\left.\mathrm{d}_{6}\right)$ $\delta 7.79(\mathrm{~d}, 2 \mathrm{H}, J=7.4 \mathrm{~Hz}, \mathrm{Ar}-\mathrm{H}$ of the Fmoc group), $7.63(\mathrm{~d}, 2 \mathrm{H}, \mathrm{J}=7.4 \mathrm{~Hz}, \mathrm{Ar}-\mathrm{H}$ of the Fmoc group), 7.39 $(\mathrm{t}, 2 \mathrm{H}, \mathrm{J}=7.4 \mathrm{~Hz}$, Ar-H of the Fmoc group), $7.31(\mathrm{t}$, $2 \mathrm{H}, J=7.4 \mathrm{~Hz}$, Ar-H of the Fmoc group), 4.40 (d, $2 \mathrm{H}$, $J=6.4 \mathrm{~Hz},=\mathrm{CH}-\mathrm{CH}_{2}-$ of the Fmoc group $), 4.20(\mathrm{t}, 1 \mathrm{H}$, $J=6.4 \mathrm{~Hz},=\mathrm{CH}-\mathrm{CH}_{2}-$ of the Fmoc group $), 3.20(\mathrm{t}, 2 \mathrm{H}$, $\left.J=6.4 \mathrm{~Hz},-\mathrm{CH}_{2}-\mathrm{CH}_{2}-\mathrm{CO}-\right), 2.91\left(\mathrm{t}, 2 \mathrm{H}, J=7.3 \mathrm{~Hz},-\mathrm{CH}_{2}-\right.$ $\mathrm{CH}_{2}-\mathrm{N}$ ), $1.77-1.83\left(\mathrm{~m}, 2 \mathrm{H},-\mathrm{CH}_{2}-\mathrm{CH}_{2}-\mathrm{CH}_{2}-\right.$ ); ${ }^{13} \mathrm{C}-$ n.m.r. $\left(\right.$ DMSO-d $\left._{6}\right) \delta 159.49,145.41,142.80,128.94,128.27,126.21$, 121.10, 67.83, 48.64, 38.42, 29.32; ESI-TOF MS calcd for $\mathrm{C}_{18} \mathrm{H}_{20} \mathrm{~N}_{2} \mathrm{O}_{2}(\mathrm{M}+\mathrm{H})^{+}$297.1603, found 297.1615; Anal. Calcd for $\mathrm{C}_{18} \mathrm{H}_{20} \mathrm{~N}_{2} \mathrm{O}_{2} \mathrm{Cl}+0.75 \mathrm{H}_{2} \mathrm{O}$ : C, 62.42; $\mathrm{H}, 6.55 ; \mathrm{N}$, 8.09, found $\mathrm{C}, 62.65 ; \mathrm{H}, 6.76$ : N, 8.61.
2.10. 9-Fluorenylmethyl 3-[4-[[4-[[4-(tert-butoxycarbonyl) amino-1-methylpyrrol-2-yl] carbonyl]amino-1-methylpyrrol2-yl]carbonyl]amino-1 - methylpyrrol - 2 - yl]carboxyl]aminopropyl-1-carbamate (18). Compounds 10 (50 mg, $0.1 \mathrm{mmol}$ ) and $17(38 \mathrm{mg}, 0.11 \mathrm{mmol})$ were dissolved in DMF $(3 \mathrm{~mL})$ and dicyclohexylcarbodiimide $(26 \mathrm{mg}, 0.12 \mathrm{mmol}$ ) and 1hydroxybenzotriazole ( $17 \mathrm{mg}, 0.12 \mathrm{mmol}$ ) were added to the solution. After stirring for 24 hours at room temperature, the solution was diluted with ethyl acetate $(50 \mathrm{~mL})$ and washed with $3 \mathrm{M} \mathrm{HCl}$ aq. $(25 \mathrm{~mL} \times 3), \mathrm{H}_{2} \mathrm{O}(25 \mathrm{~mL}), 5 \% \mathrm{NaHCO}_{3}$ aq. $(25 \mathrm{~mL} \times 2)$, and $\mathrm{H}_{2} \mathrm{O}(25 \mathrm{~mL})$. The organic layer was dried over anhydrous magnesium sulfate, evaporated to dryness, and the residue was subjected to silica gel column chromatography using a methanol/chloroform (1: 49, v/v) solvent system to give 18 (75.6 mg, 96\% yield); ${ }^{1} \mathrm{H}$-n.m.r. (DMSO-d 6 ) $\delta 9.87$ (s, 1H, -NHCO-), 9.85 (s, 1H, -NHCO-), $9.08(\mathrm{~s}, 1 \mathrm{H}, \mathrm{Boc}-\mathrm{NH}), 7.95\left(\mathrm{t}, 1 \mathrm{H}, J=5.5 \mathrm{~Hz},-\mathrm{CH}_{2} \mathrm{NHCO}-\right.$ ), $7.88(\mathrm{~d}, 2 \mathrm{H}, J=7.4 \mathrm{~Hz}, \mathrm{Ar}-\mathrm{H}$ of the Fmoc group), $7.69(\mathrm{~d}, 2 \mathrm{H}, \mathrm{J}=7.4 \mathrm{~Hz}, \mathrm{Ar}-\mathrm{H}$ of the Fmoc group), 7.41 $(\mathrm{t}, 2 \mathrm{H}, J=7.4 \mathrm{~Hz}, \mathrm{Ar}-\mathrm{H}$ of the Fmoc group), $7.32(\mathrm{t}, 2 \mathrm{H}$, $J=7.4 \mathrm{~Hz}$, Ar-H of the Fmoc group), $7.26(\mathrm{t}, 1 \mathrm{H}, J=5.6 \mathrm{~Hz}$, $\left.-\mathrm{CH}_{2} \mathrm{NH}-\mathrm{Fmoc}\right), 7.21$ (d, $\left.1 \mathrm{H}, J=1.7 \mathrm{~Hz}, \mathrm{Py}-\mathrm{H}\right), 7.18$ (d, $1 \mathrm{H}, J=1.5 \mathrm{~Hz}, \mathrm{Py}-\mathrm{H}), 7.04$ (d, 1H, J = 1.7 Hz, Py-H), 6.89 (s, 1H, Py-H), 6.86 (d, 1H, J = 1.5 Hz, Py-H), $6.84(\mathrm{~s}, 1 \mathrm{H}$, Py-H), $4.32\left(\mathrm{~d}, 2 \mathrm{H}, \mathrm{J}=6.6 \mathrm{~Hz},=\mathrm{CH}-\mathrm{CH}_{2}-\right.$ of the Fmoc group), $4.22\left(\mathrm{t}, 1 \mathrm{H}, \mathrm{J}=6.6 \mathrm{~Hz},=\mathrm{CH}-\mathrm{CH}_{2}-\right.$ of the $\mathrm{Fmoc}$ group), 3.84 (s, 3H, Py- $\left.\mathrm{CH}_{3}\right), 3.81$ (s, $\left.3 \mathrm{H}, \mathrm{Py}_{-} \mathrm{CH}_{3}\right), 3.80$ (s, $\left.3 \mathrm{H}, \mathrm{Py}_{-} \mathrm{CH}_{3}\right), 3.17\left(\mathrm{~m}, 2 \mathrm{H},-\mathrm{NHCH}_{2} \mathrm{CH}_{2}-\right), 3.03(\mathrm{~m}, 2 \mathrm{H}$, $\left.-\mathrm{CH}_{2} \mathrm{CH}_{2} \mathrm{NH}-\right), 1.62\left(\mathrm{~m}, 2 \mathrm{H},-\mathrm{CH}_{2} \mathrm{CH}_{2} \mathrm{CH}_{2}-\right), 1.46(\mathrm{~s}, 9 \mathrm{H}$, $-\mathrm{CH}_{3}$ of the Boc group); ${ }^{13} \mathrm{C}$-n.m.r. (DMSO-d $\left.{ }_{6}\right) \delta 161.25$, $158.45,158.41,156.10,152.80,143.91,140.72,127.56$, $127.02,125.09,122.89,122.82,122.73,122.29,122.15$, $120.08,118.34,117.77,117.02,104.68,104.49,103.81,78.35$, $65.19,46.76,38.03,36.00,35.89,29.64$; ESI-TOF MS calcd for $\mathrm{C}_{41} \mathrm{H}_{47} \mathrm{~N}_{8} \mathrm{O}_{7}(\mathrm{M}+\mathrm{H})^{+}$763.3568, found 763.3591; Anal. Calcd for $\mathrm{C}_{41} \mathrm{H}_{46} \mathrm{~N}_{8} \mathrm{O}_{7}+2 \mathrm{H}_{2} \mathrm{O}$ : C, 61.64; $\mathrm{H}, 6.31 ; \mathrm{N}, 14.03$, found $\mathrm{C}, 62.12 ; \mathrm{H}, 6.10 ; \mathrm{N}, 14.09$.

2.11.9-Fluorenylmethyl 3-[4-[[4-[(4-Formylamino-1-methylpyrrol-2-yl)carbonyl]amino-1 - methylpyrrol - 2 - yl]carbonyl] amino-1-methylpyrrol-2 -yl]carboxyl]aminopropyl-1-carbamate (19). Compound 18 (0.696 g, $0.91 \mathrm{mmol})$ was dissolved in methanol $(12 \mathrm{~mL})$ and acetyl chloride $(3 \mathrm{~mL}$, $42 \mathrm{mmol}$ ) was added to the solution at $0^{\circ} \mathrm{C}$. After stirring for 30 minutes, the reaction solution was concentrated in vacuo to dryness. The residue was dissolved in methanol $(5 \mathrm{~mL}) / \mathrm{THF}(10 \mathrm{~mL})$ and $N$-formylimidazole, prepared by the treatment of formic acid $(41 \mu \mathrm{L}, 1.09 \mathrm{mmol})$ with $1,1^{\prime}$-carbonyldiimidazole $(177 \mathrm{mg}, 1.09 \mathrm{mmol})$ in THF $(4 \mathrm{~mL})$ at room temperature for 5 minutes [11], was added to the solution at $-40^{\circ} \mathrm{C}$. After stirring at $-40^{\circ} \mathrm{C}$ for 1 hour, the solution was diluted with $5 \% \mathrm{NaHCO}_{3}$ aq. $(10 \mathrm{~mL})$, washed with ethyl acetate $(10 \mathrm{~mL} \times 2)$, and neutralized with $10 \% \mathrm{HCl}$ aq. to $\mathrm{ca}$. $\mathrm{pH} \mathrm{2}$. Compound 10 was extracted with ethyl acetate $(20 \mathrm{~mL} \times 3)$ from the resulting solution. The organic layer was washed with $\mathrm{H}_{2} \mathrm{O}(30 \mathrm{~mL})$, dried over anhydrous magnesium sulfate, and evaporated to dryness. The residue was subjected to silica gel column 
chromatography using a methanol/chloroform (1:19, v/v) solvent system. Compound 19 was obtained in $88 \%$ yield $(0.555 \mathrm{~g}) ;{ }^{1} \mathrm{H}$-n.m.r. (DMSO-d 6$) \delta 10.04(\mathrm{~d}, 1 \mathrm{H}, J=1.7 \mathrm{~Hz}$, -NHCHO), 9.91 (s, 1H, -NHCO-), 9.88 (s, 1H, -NHCO-), $8.13(\mathrm{~d}, 1 \mathrm{H}, J=1.7 \mathrm{~Hz},-\mathrm{NHCHO}), 7.96(\mathrm{t}, 1 \mathrm{H}, J=5.6 \mathrm{~Hz}$, $\left.-\mathrm{CH}_{2} \mathrm{NHCO}-\right), 7.88$ (d, 2H, J $=7.4 \mathrm{~Hz}, \mathrm{Ar}-\mathrm{H}$ of the Fmoc group), 7.65 (d, $2 \mathrm{H}, J=7.4 \mathrm{~Hz}, \mathrm{Ar}-\mathrm{H}$ of the Fmoc group), $7.41(\mathrm{t}, 2 \mathrm{H}, J=7.4 \mathrm{~Hz}$, Ar-H of the Fmoc group), $7.33(\mathrm{t}, 2 \mathrm{H}$, $J=7.4 \mathrm{~Hz}$, Ar-H of the Fmoc group), $7.25(\mathrm{t}, 1 \mathrm{H}, J=5.6 \mathrm{~Hz}$, $-\mathrm{CH}_{2} \mathrm{NH}-\mathrm{Fmoc}$ ), 7.23 (d, $\left.1 \mathrm{H}, J=1.8 \mathrm{~Hz}, \mathrm{Py}-\mathrm{H}\right), 7.20(\mathrm{~d}$, $1 \mathrm{H}, J=1.8 \mathrm{~Hz}, \mathrm{Py}-\mathrm{H}), 7.19(\mathrm{~d}, 1 \mathrm{H}, J=1.5 \mathrm{~Hz}, \mathrm{Py}-\mathrm{H}), 7.04$ $(\mathrm{d}, 1 \mathrm{H}, J=1.8 \mathrm{~Hz}, \mathrm{Py}-\mathrm{H}), 6.92(\mathrm{~d}, 1 \mathrm{H}, J=1.8 \mathrm{~Hz}, \mathrm{Py}-\mathrm{H})$, $6.87(\mathrm{~d}, 1 \mathrm{H}, J=1.5 \mathrm{~Hz}, \mathrm{Py}-\mathrm{H}), 4.32(\mathrm{~d}, 2 \mathrm{H}, J=6.7 \mathrm{~Hz}$, $=\mathrm{CH}-\mathrm{CH}_{2}-$ of the Fmoc group), $4.22(\mathrm{t}, 1 \mathrm{H}, J=6.7 \mathrm{~Hz}$, $=\mathrm{CH}-\mathrm{CH}_{2}-$ of the Fmoc group), $3.85\left(\mathrm{~s}, 6 \mathrm{H}, \mathrm{Py}_{-} \mathrm{CH}_{3} \times 2\right)$, $3.80\left(\mathrm{~s}, 3 \mathrm{H}, \mathrm{Py}-\mathrm{CH}_{3}\right), 3.16\left(\mathrm{~m}, 2 \mathrm{H},-\mathrm{NHCH}_{2} \mathrm{CH}_{2}-\right), 3.03$ (m, $\left.2 \mathrm{H},-\mathrm{CH}_{2} \mathrm{CH}_{2} \mathrm{NH}-\right), 1.61\left(\mathrm{~m}, 2 \mathrm{H},-\mathrm{CH}_{2} \mathrm{CH}_{2} \mathrm{CH}_{2}-\right)$; ${ }^{13}$ C-n.m.r. (DMSO-d 6 ) $\delta 161.24,158.42,158.27,157.85$, $143.91,140.72,128.89,127.56,127.03,125.09,122.95$, $122.76,122.05,120.71,120.08,118.34,117.77,104.63$, 104.08, 103.93, 65.19, 46.76, 38.88, 38.03, 36.09, 35.89; ESI-TOF MS calcd for $\mathrm{C}_{37} \mathrm{H}_{39} \mathrm{~N}_{8} \mathrm{O}_{6}(\mathrm{M}+\mathrm{H})^{+}$691.2993, found 691.2974; Anal. Calcd for $\mathrm{C}_{37} \mathrm{H}_{38} \mathrm{~N}_{8} \mathrm{O}_{6}+\mathrm{H}_{2} \mathrm{O}$ : C, 62.70; H, 5.69; N, 15.81, found C, 63.05; H, 5.69; N, 16.00 .

2.12. $N^{2}-[3-[[4-[[4-[(4-F o r m y l a m i n o-1-m e t h y l p y r r o l-2-y l)$ carbonyl]amino-1-methylpyrrol- 2 - yl]carbonyl] amino-1-methylpyrrol-2-yl] carboxyl] aminopropyl]-3',5' -di-O-acetyl-O ${ }^{6}$ [2-(4-nitrophenyl)ethyl]-2'-deoxyguanosine (21). Compound 19 (150 mg. $0.22 \mathrm{mmol}$ ) was dissolved in $10 \%$ piperidine/DMF $(1 \mathrm{~mL})$. After stirring at room temperature for 20 minutes, the solution was concentrated in vacuo to dryness. The residue and $3^{\prime}, 5^{\prime}$-di-O-acetyl-2-fluoro$\mathrm{O}^{6}$-[2-(4-nitrophenyl)ethyl]-2' -deoxyinosine (20) (0.219 g, $0.43 \mathrm{mmol}$ ) were dissolved in DMF $(2 \mathrm{~mL})$ and triethylamine $(60 \mu \mathrm{L}, 0.43 \mathrm{mmol})$ was added to the solution. After stirring at $60^{\circ} \mathrm{C}$ for 12 hours, the reaction solution was concentrated in vacuo to dryness. The residue was subjected to silica gel column chromatography using a methanol/chloroform (1: 19, v/v) solvent system to give $21(0.168 \mathrm{~g}, 81 \%$ yield); ${ }^{1} \mathrm{H}$-n.m.r. (DMSO-d 6 ) $\delta 10.19$ (d, $1 \mathrm{H}, J=1.7 \mathrm{~Hz}$, -NHCHO), 9.91 (s, 1H, -NHCO-), 9.87 (s, 1H, -NHCO-), $8.16(\mathrm{~d}, 2 \mathrm{H}, J=8 \mathrm{~Hz}, \mathrm{Ar}-\mathrm{H}$ of the NPE group), $8.13(\mathrm{~d}$, $1 \mathrm{H}, J=1.7 \mathrm{~Hz},-\mathrm{NHCHO}), 8.03(\mathrm{~s}, 1 \mathrm{H}, \mathrm{H}-8), 8.01(\mathrm{t}, 1 \mathrm{H}$, $\left.J=5.4 \mathrm{~Hz},-\mathrm{CH}_{2} \mathrm{NHCO}-\right), 7.60(\mathrm{~d}, 2 \mathrm{H}, J=8 \mathrm{~Hz}, \mathrm{Ar}-\mathrm{H}$ of the NPE group), $7.22(\mathrm{~d}, 1 \mathrm{H}, J=1.7 \mathrm{~Hz}, \mathrm{Py}-\mathrm{H}), 7.19(\mathrm{~d}, 1 \mathrm{H}$, $J=1.8 \mathrm{~Hz}, \mathrm{Py}-\mathrm{H}), 7.16(\mathrm{~d}, 1 \mathrm{H}, J=1.7 \mathrm{~Hz}, \mathrm{Py}-\mathrm{H}), 7.04(\mathrm{~d}$, $1 \mathrm{H}, J=1.7 \mathrm{~Hz}, \mathrm{Py}-\mathrm{H}), 7.01\left(\mathrm{t}, 1 \mathrm{H}, J=5.7 \mathrm{~Hz}, \mathrm{~N}^{2}-\mathrm{H}\right), 6.91$ $(\mathrm{d}, 1 \mathrm{H}, J=1.9 \mathrm{~Hz}, \mathrm{Py}-\mathrm{H}), 6.90(\mathrm{~d}, 1 \mathrm{H}, J=1.3 \mathrm{~Hz}, \mathrm{Py}-\mathrm{H})$, $6.24\left(\mathrm{t}, 1 \mathrm{H}, J=6.7 \mathrm{~Hz}, \mathrm{H}-1^{\prime}\right), 5.41\left(\mathrm{~m}, 1 \mathrm{H}, \mathrm{H}-3^{\prime}\right), 4.68(\mathrm{t}$, $J=6.6 \mathrm{~Hz},-\mathrm{CH}_{2} \mathrm{CH}_{2}-$ of the NPE group), $4.31(\mathrm{~m}, 1 \mathrm{H}$, $\left.\mathrm{H}-4^{\prime}\right), 4.19\left(\mathrm{~m}, 2 \mathrm{H}, \mathrm{H}-5^{\prime}\right.$ and $\left.5^{\prime \prime}\right), 3.84\left(\mathrm{~s}, 6 \mathrm{H}, \mathrm{Py}-\mathrm{CH}_{3} \times 2\right)$, $3.80\left(\mathrm{~s}, 3 \mathrm{H}, \mathrm{Py}_{-} \mathrm{CH}_{3}\right), 3.36\left(\mathrm{~m}, 2 \mathrm{H},-\mathrm{NHCH}_{2} \mathrm{CH}_{2}-\right), 3.25$ $\left(\mathrm{m}, 4 \mathrm{H},-\mathrm{CH}_{2} \mathrm{CH}_{2}\right.$ - of the NPE group and $-\mathrm{CH}_{2} \mathrm{CH}_{2} \mathrm{NH}-$ ), $3.12\left(\mathrm{~m}, 1 \mathrm{H}, \mathrm{H}-2^{\prime}\right), 2.45\left(\mathrm{~m}, 1 \mathrm{H}, \mathrm{H}-2^{\prime \prime}\right), 2.08(\mathrm{~s}, 3 \mathrm{H}$, the Ac group), 1.99 ( $\mathrm{s}, 3 \mathrm{H}$, the Ac group), $1.76(\mathrm{~m}, 2 \mathrm{H}$, $-\mathrm{CH}_{2} \mathrm{CH}_{2} \mathrm{CH}_{2-}$ ); ${ }^{13} \mathrm{C}-$ n.m.r. (DMSO-d ${ }_{6}$ ) $\delta$ 170.10, 169.99, $161.31,159.99,158.70,158.42,158.28,157.86,146.71$
$146.22,130.20,123.38,122.95,122.77,122.16,120.72$, 118.37, 117.72, 104.64, 104.13, 103.94, 81.35, 65.41, 63.64, $36.22,36.04,35.89,34.31,20.77,20.47$; ESI-TOF MS calcd for $\mathrm{C}_{44} \mathrm{H}_{50} \mathrm{~N}_{13} \mathrm{O}_{12}(\mathrm{M}+\mathrm{H})^{+}$952.3702, found 952.3738 .

2.13. $N^{2}-[3-[[4-[[4-[(4-F o r m y l a m i n o-1-m e t h y l p y r r o l-2-y l)$ carbonyl]amino-1-methylpyrrol-2 - yl]carbonyl]amino-1-methylpyrrol - 2 - yl]carboxyl]aminopropyl] - 2' - deoxyguanosine (Hybrid 3). Compound 21 (0.116 g, $0.12 \mathrm{mmol})$ was treated with $0.5 \mathrm{M} \mathrm{DBU} /$ pyridine $(2 \mathrm{~mL})$. After stirring at room temperature for 12 hours, ammonium chloride $(80 \mathrm{mg}$, $1.5 \mathrm{mmol}$ ) was added to the solution. The solution was diluted with chloroform $(60 \mathrm{~mL})$ and washed with $\mathrm{H}_{2} \mathrm{O}$ $(25 \mathrm{~mL})$. The organic layer was dried over anhydrous magnesium sulfate, evaporated to dryness, and the residue was subjected to silica gel column chromatography using a methanol/chloroform (1: 4, v/v) solvent system to give $N^{2}$-[3-[[4-[[4-[(4-formylamino-1-methylpyrrol-2-yl)carbonyl] amino- 1 -methylpyrrol-2-yl] carbonyl] amino-1-methylpyrrol-2-yl] carboxyl] aminopropyl] -3' , $^{\prime}$-di-O-acetyl-2' -deoxyguanosine $\left(71 \mathrm{mg}, 73 \%\right.$ yield); ${ }^{1} \mathrm{H}$-n.m.r. $\left(\mathrm{DMSO}^{\left.-\mathrm{d}_{6}\right)} \delta\right.$ $10.70\left(\mathrm{br}, 1 \mathrm{H}, \mathrm{N}^{1}-\mathrm{H}\right), 10.07$ (d, $\left.1 \mathrm{H}, J=1.5 \mathrm{~Hz},-\mathrm{NHCHO}\right)$, 9.91 (s, 1H, -NHCO-), 9.87 (s, 1H, -NHCO-), 8.12 (d, $1 \mathrm{H}, J=1.5 \mathrm{~Hz},-\mathrm{NHCHO}), 8.04(\mathrm{t}, 1 \mathrm{H}, J=5.8 \mathrm{~Hz}$, $-\mathrm{CH}_{2} \mathrm{NHCO}-$ ), 7.87 (s, $\left.1 \mathrm{H}, \mathrm{H}-8\right), 7.22(\mathrm{~d}, 1 \mathrm{H}, \mathrm{J}=1.6 \mathrm{~Hz}$, Py-H), $7.19(\mathrm{~d}, 1 \mathrm{H}, J=1.6 \mathrm{~Hz}, \mathrm{Py}-\mathrm{H}), 7.17(\mathrm{~d}, 1 \mathrm{H}$, $J=1.6 \mathrm{~Hz}, \mathrm{Py}-\mathrm{H}), 7.06(\mathrm{~d}, 1 \mathrm{H}, J=1.7 \mathrm{~Hz}, \mathrm{Py}-\mathrm{H}), 6.91(\mathrm{~d}$, $1 \mathrm{H}, J=1.7 \mathrm{~Hz}, \mathrm{Py}-\mathrm{H}), 6.90(\mathrm{~d}, 1 \mathrm{H}, J=1.6 \mathrm{~Hz}, \mathrm{Py}-\mathrm{H}), 6.64$ $\left(\mathrm{t}, 1 \mathrm{H}, J=5 \mathrm{~Hz}, \mathrm{~N}^{2}-\mathrm{H}\right), 6.18\left(\mathrm{t}, 1 \mathrm{H}, J=7 \mathrm{~Hz}, \mathrm{H}-1^{\prime}\right), 5.46$ $\left(\mathrm{m}, 1 \mathrm{H}, \mathrm{H}-3^{\prime}\right), 4.30\left(\mathrm{~m}, 1 \mathrm{H}, \mathrm{H}-4^{\prime}\right), 4.17$ (m, 2H, H-5' and $\left.5^{\prime \prime}\right), 3.84\left(\mathrm{~s}, 6 \mathrm{H}, \mathrm{Py}_{-} \mathrm{CH}_{3} \times 2\right), 3.80\left(\mathrm{~s}, 3 \mathrm{H}, \mathrm{Py}_{-} \mathrm{CH}_{3}\right), 3.35(\mathrm{~m}$, $\left.2 \mathrm{H},-\mathrm{NHCH}_{2} \mathrm{CH}_{2}-\right), 3.25\left(\mathrm{~m}, 2 \mathrm{H},-\mathrm{CH}_{2} \mathrm{CH}_{2} \mathrm{NH}-\right)$, 3.05 (m, $\left.1 \mathrm{H}, \mathrm{H}-2^{\prime}\right), 2.47$ (m, 1H, H-2' $), 2.08$ (s, 3H, the Ac group), 1.99 (s, 3H, the Ac group), $1.74\left(\mathrm{~m}, 2 \mathrm{H},-\mathrm{CH}_{2} \mathrm{CH}_{2} \mathrm{CH}_{2}-\right.$ ); ESI-TOF MS calcd for $\mathrm{C}_{36} \mathrm{H}_{43} \mathrm{~N}_{12} \mathrm{O}_{10}(\mathrm{M}+\mathrm{H})^{+}$803.3225, found 803.3201 .

$N^{2}$-[3-[[4-[ [4-[(4-Formylamino- 1 -methylpyrrol- 2 -yl) carbonyl ] amino - 1 - methylpyrrol -2-yl] carbonyl] amino-1methylpyrrol-2-yl] carboxyl] aminopropyl]-3', 5' -di- $O$ - acetyl-2'-deoxyguanosine $(71.4 \mathrm{mg}, 0.089 \mathrm{mmol})$ was dissolved in pyridine $(0.2 \mathrm{~mL})$ and $0.05 \mathrm{M}$ sodium methoxide/methanol $(0.8 \mathrm{~mL})$ was added to the solution. After stirring at room temperature for 2 hours, the solution was neutralized with Dowex-50WX2 ion-exchange resin $\left(\mathrm{H}^{+}\right.$ form) after which time the resin was immediately removed by filtration. The filtrate was evaporated to dryness and the residue was crystallized from methanol. The resulting white crystals were collected by vacuum filtration to give Hybrid 3 (51.1 mg, 80\% yield); ${ }^{1} \mathrm{H}-$ n.m.r. $\left(\mathrm{DMSO}_{-} \mathrm{d}_{6}\right) \delta 10.80$ (br, $1 \mathrm{H}$, $\left.\mathrm{N}^{1}-\mathrm{H}\right), 10.05$ (d, $\left.1 \mathrm{H}, J=1.6 \mathrm{~Hz},-\mathrm{NHCHO}\right), 9.91(\mathrm{~s}, 1 \mathrm{H}$, -NHCO-), 9.87 (s, 1H, -NHCO-), 8.12 (m, 2H, -NHCHO and $\mathrm{H}-8), 8.07$ (t, $\left.1 \mathrm{H}, J=5.6 \mathrm{~Hz},-\mathrm{CH}_{2} \mathrm{NHCO}-\right), 7.22(\mathrm{~d}$, $1 \mathrm{H}, J=1.8 \mathrm{~Hz}, \mathrm{Py}-\mathrm{H}), 7.19(\mathrm{~d}, 1 \mathrm{H}, J=1.8 \mathrm{~Hz}, \mathrm{Py}-\mathrm{H}), 7.18$ $(\mathrm{d}, 1 \mathrm{H}, J=1.8 \mathrm{~Hz}, \mathrm{Py}-\mathrm{H}), 7.04(\mathrm{~d}, 1 \mathrm{H}, J=1.8 \mathrm{~Hz}, \mathrm{Py}-\mathrm{H})$, $6.91(\mathrm{~d}, 1 \mathrm{H}, J=1.8 \mathrm{~Hz}, \mathrm{Py}-\mathrm{H}), 6.89$ (d, $1 \mathrm{H}, J=1.8 \mathrm{~Hz}, \mathrm{Py}-$ $\mathrm{H}), 6.62\left(\mathrm{br}, 1 \mathrm{H}, \mathrm{N}^{2}-\mathrm{H}\right), 6.17\left(\mathrm{t}, 1 \mathrm{H}, J=6.8 \mathrm{~Hz}, \mathrm{H}-1^{\prime}\right), 4.36$ $\left(\mathrm{m}, 1 \mathrm{H}, \mathrm{H}-3^{\prime}\right), 3.84$ (s, 3H, Py-CH $\mathrm{CH}_{3}, 3.83$ (s, 3H, $\mathrm{Py}-\mathrm{CH}_{3}$ ), $3.82\left(\mathrm{~m}, 1 \mathrm{H}, \mathrm{H}-4^{\prime}\right), 3.80\left(\mathrm{~s}, 3 \mathrm{H}, \mathrm{Py}-\mathrm{CH}_{3}\right), 3.56(\mathrm{~m}, 1 \mathrm{H}$, 
$\left.\mathrm{H}-5^{\prime}\right), 3.50\left(\mathrm{~m}, 1 \mathrm{H}, \mathrm{H}-5^{\prime \prime}\right), 3.33\left(\mathrm{~m}, 2 \mathrm{H},-\mathrm{NHCH}_{2} \mathrm{CH}_{2}-\right)$, $3.25\left(\mathrm{~m}, 2 \mathrm{H},-\mathrm{CH}_{2} \mathrm{CH}_{2} \mathrm{NH}-\right)$, $2.61\left(\mathrm{~m}, 1 \mathrm{H}, \mathrm{H}-2^{\prime}\right), 2.24$ (m, 1H, H-2 $2^{\prime \prime}$ ), $1.75\left(\mathrm{~m}, 2 \mathrm{H},-\mathrm{CH}_{2} \mathrm{CH}_{2} \mathrm{CH}_{2-}\right)$; ${ }^{13} \mathrm{C}-$ n.m.r. $\left(\mathrm{DMSO}_{-} \mathrm{d}_{6}\right) \delta 161.43,158.43,158.23,157.85,156.17,152.78$, $150.19,135.69,122.94,122.88,122.75,122.13,122.05$, $120.71,118.37,117.82,104.65,104.65,104.23,103.95$, 87.71, 83.16, 70.65, 61.65, 38.21, 36.09, 36.05, 35.90. 29.15; ESI-TOF MS calcd for $\mathrm{C}_{32} \mathrm{H}_{39} \mathrm{~N}_{12} \mathrm{O}_{8}(\mathrm{M}+\mathrm{H})^{+} 719.3014$, found 719.3005; Anal. Calcd for $\mathrm{C}_{37} \mathrm{H}_{38} \mathrm{~N}_{8} \mathrm{O}_{6}+1.5 \mathrm{H}_{2} \mathrm{O}$ : C, 51.54; H, 5.54; N, 22.54, found C, 51.36; H, 5.50; N, 22.26.

2.14. Circular Dichroism (CD) Spectroscopy. CD spectra were measured using a JASCO J-720 spectropolarimeter equipped with a thermoelectrically controlled cell holder and a cuvette with a path length of $10 \mathrm{~mm}$. CD titrations were conducted at $20^{\circ} \mathrm{C}$ by incrementally injecting $15 \mu \mathrm{L}$ aliquots of a $270 \mu \mathrm{M}$ ligand (Hybrid 2, Hybrid 3, or Dst) solution into $3 \mathrm{~mL}$ of a $5.8 \mu \mathrm{M}$ duplex [DNA 1: d- $\left(5^{\prime}-\right.$ CGCAAATTGGC-3' $\left.{ }^{\prime}\right)-\mathrm{d}-\left(3^{\prime}\right.$-GCGTTTAACCG-5' $\left.5^{\prime}\right)$, DNA 2: d- $\left(5^{\prime}\right.$-CGCAGACTGGC-3')-d-( $3^{\prime}$-GCGTCTGACCG$\left.5^{\prime}\right)$, or DNA 3: d-(5'-GCACGACTACG-3')-d- $\left(3^{\prime}-\right.$ CGTGCTGATGC- $\left.\left.5^{\prime}\right)\right]$ solution in the same buffer $[19,20]$. The resulting ligand concentration in the DNA duplex solution increased from 0 to $18.1 \mu \mathrm{M}$. After each injection, the CD spectrum was recorded between 220 and $380 \mathrm{~nm}$.

2.15. UV Absorption Spectroscopy. In an effort to assess the effect of the bound ligand (Hybrid 1, Hybrid 2, Hybrid 3, or Dst) on the thermal stability of the three duplexes (DNA 13), UV melting experiments were conducted in the absence and presence of each ligand. Absorbance versus temperature profiles were measured using a Shimazu TMSPC-8/UV-1600 spectrophotometer equipped with a thermoelectrically controlled cell holder at $260 \mathrm{~nm}$ and a heating rate of $1.0^{\circ} \mathrm{C} / \mathrm{min}$. The concentration of each 11 mer duplex was $4.3 \mu \mathrm{M}$, while the ligand concentrations varied from 0 to $12.9 \mu \mathrm{M}$.

\section{Results and Discussion}

Hybrid 2 incorporating the 3-aminopropionyl linker was synthesized by condensation of $N^{2}$-(Fmoc- $\beta$-alanyl)- $3^{\prime}, 5^{\prime}$ $O$-TIPDS-guanosine (12) with pyrrole amide trimer 10 as shown in Scheme 2.

Pyrrole amide trimer $\mathbf{1 0}$ was prepared using modifications of the method of Baird and Dervan [3]. The monomer building blocks methyl 4-nitropyrrole-2-carboxylate (5) and 4-(tert-butoxycarbonyl)amino-1-methylpyrrole-2-carboxylic acid (6) were synthesized from $N$-methylpyrrole via 2-trichloroacetylation, 4-nitration and esterification to give 5 , and subsequent alkaline hydrolysis of ester 5 to give $\mathbf{6}$. Using pyrrole monomers 5 and $\mathbf{6}$, pyrroleamide trimer $\mathbf{1 0}$ was synthesized as shown in Scheme 1. Monomer 5 was reduced to give methyl 4-aminopyrrole-2-carboxylate, which was then coupled with 6 in the presence of 1-ethyl-3-(3dimethylaminopropyl)carbodiimide hydrochloride (EDCI) and 4-(dimethylamino)pyridine (DMAP) to give dimer 7 . Next, following deprotection of the Boc group, dimer 7 was coupled with $\mathbf{6}$ to give trimer $\mathbf{8}$. Trimer $\mathbf{8}$ was then converted to trimer 10 through alkaline hydrolysis of the ester moiety, deprotection of the Boc group, and finally $N$-formylation [11].

We initially attempted the synthesis of the pyrrolepolyamide-2'-deoxyguanosine hybrid (Hybrid 2) by condensation of $3^{\prime}, 5^{\prime}$-O-TIPDS-guanosine (12) and pyrrole amide trimer 11, prepared by coupling pyrrole amide trimer 9 with ethyl $\beta$-alanine, alkaline hydrolysis of the ester moiety, deprotection of the Boc group, and finally $N$-formylation. The desired compound was not obtained when using EDCI/DMAP or dicyclohexylcarbodiimide (DCC)/hydroxybenztriazole (HOBT) as condensation reagents due to the low reactivity of the exocyclic amino group of the guanine base. We then attempted the synthesis of Hybrid 2 using pyrrole amide trimer 10 and $N^{2}$-[3(9-fluorenylmethoxycarbonylamino)propioyl]-3', $5^{\prime}-\mathrm{O}-$

TIPDS-guanosine (13). Compound 13 was prepared by reaction of $3^{\prime}, 5^{\prime}$-O-TIPDS-guanosine (12) with 3-(Fmocamino)propionyl chloride, prepared from $N$-Fmoc- $\beta$ alanine and $\mathrm{SOCl}_{2}$, in $43 \%$ yield. Treatment of 13 with pyrroleamide trimer $\mathbf{1 0}$ in the presence of EDCI and a large excess of DMAP generated pyrrolepolyamide2 -deoxyguanosine derivative $\mathbf{1 4}$ in $83 \%$ yield through deprotection of the Fmoc group of $\mathbf{1 3}$ and condensation of the amine product with 10. Hybrid 2 was obtained in quantitative yield from 14 by treatment with $0.2 \mathrm{M}$ tetraethylammonium fluoride (TEAF) in THF.

Hybrid 3 incorporating the 3 -aminopropyl linker was synthesized using $3^{\prime}, 5^{\prime}$-di-O-acetyl-2-fluoro- $\mathrm{O}^{6}$-[2-(4nitrophenyl)ethyl]-2' -deoxyinosine (20) and pyrrole amide trimer 19 as shown in Scheme 4. Monoprotected propane1,3-diamine containing the Fmoc group, which can be deprotected under mild basic conditions and is stable during deprotection of the Boc group, was designed as the linker reagent. Monoprotection of propane-1,3-diamine by treatment with 9-fluorenylmethyl chloroformate (Fmoc-Cl) under basic conditions in the presence of a large excess of propane-1,3-diamine and $\mathrm{Na}_{2} \mathrm{CO}_{3}$ was initially examined. The desired compound was not obtained due to the low selectivity of monoprotection and basic lability of the Fmoc group. Pittelkov et al. reported on the monoprotection of alkanediamines $\left[\mathrm{H}_{2} \mathrm{~N}-\left(\mathrm{CH}_{2}\right) n-\mathrm{NH}_{2}: n=0-4\right]$ with Boc, $\mathrm{Cbz}$ or allyloxycarbonyl groups using the corresponding phenyl carbonates. Monoprotected alkanediamines were obtained in $46 \%-86 \%$ yield [27]. Using a modification of the method of Pittelkov et al., we then treated propane-1,3-diamine with 9-fluorenylmethyl phenyl carbonate (16), prepared from Fmoc-Cl and sodium phenoxide in $87 \%$ yield, and then $1 \mathrm{M}$ $\mathrm{HCl}$ aq. to give the $\mathrm{HCl}$ salt of 3-(Fmoc-amino)propylamine as shown in Scheme 3. Linker reagent 17 was obtained in 69\% yield. Pyrrole amide trimer 10 was condensed with linker reagent 17 in the presence of DCC, HOBT, and diisopropylethylamine (DIEA) in DMF to generate pyrrole amide trimer 18, obtained in 96\% yield. Pyrrole amide trimer 18 was then subjected to deprotection of the Boc group and subsequent $N$-formylation to give pyrrole amide trimer 19. Treatment of pyrrole amide trimer 19 with 2 -deoxy-2-fluoroinosine derivative 20 in the presence of triethylamine in DMF at $60^{\circ} \mathrm{C}$ for 12 hours generated the 


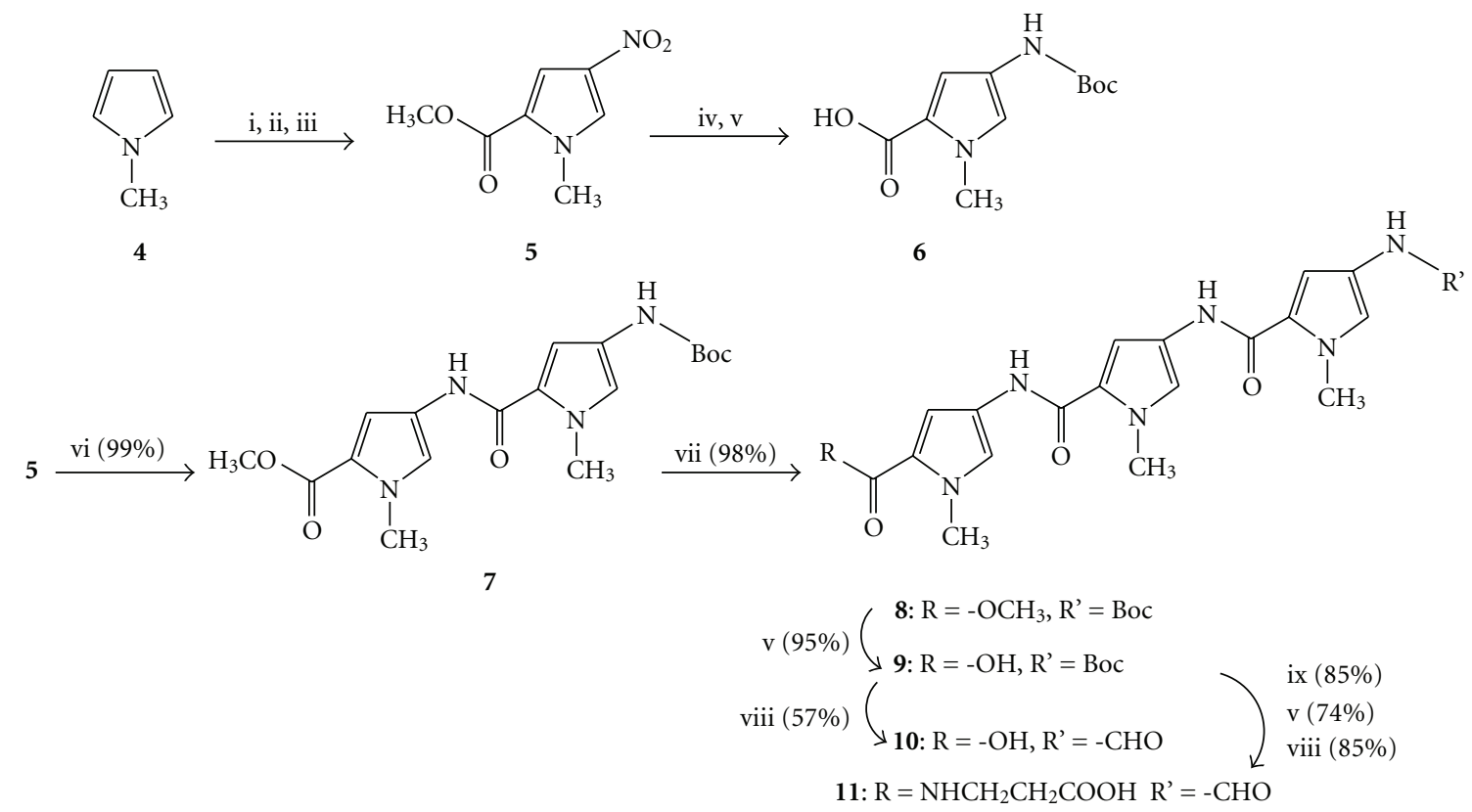

Scheme 1: Reaction conditions (i) $\mathrm{CL}_{3} \mathrm{CCOCL}, \mathrm{Et}_{2} \mathrm{O}$, rt, 1 hour. (ii) $\mathrm{HNO}_{3}, \mathrm{Ac}_{2} \mathrm{O},-10^{\circ} \mathrm{C} \sim \mathrm{rt}, 1$ hour. (iii) $\mathrm{NaOMe}, \mathrm{MeOH}, \mathrm{rt}, 15$ minutes. (iv) $1 . \mathrm{H}_{2}, 10 \% \mathrm{Pd} / \mathrm{C}$, AcOEt, rt, 12 hours; 2. (Boc) ${ }_{2} \mathrm{O}, \mathrm{Et}_{2} \mathrm{O}, \mathrm{rt}, 2$ hours. (v) $1.1 \mathrm{M} \mathrm{NaOH}, \mathrm{H}_{2} \mathrm{O} / \mathrm{MeOH}, 60^{\circ} \mathrm{C}, 1 \sim 2$ hours; $2.10 \% \mathrm{HCL}$ aq. (vi) $1 . \mathrm{H}_{2}, 10 \% \mathrm{Pd} / \mathrm{C}$, AcOEt, rt, 12 hours; 2. 6, EDCI, DMAP, DMF, rt, 3 hours. (vii) 1. AcCL, MeOH, $0^{\circ} \mathrm{C}, 30$ minutes. 2. 6, EDCI, DMAP, DMF, rt, 3 hours. (viii) 1 . AcCL, MeOH, $0^{\circ} \mathrm{C}, 30$ minutes; $2 \mathrm{HCOOH}, \mathrm{CDI}$, THF, $-40^{\circ} \mathrm{C}, 30$ minutes. (ix) ethyl $\beta$-alanine, EDCI, DMAP, DMF, rt, 3 hours.

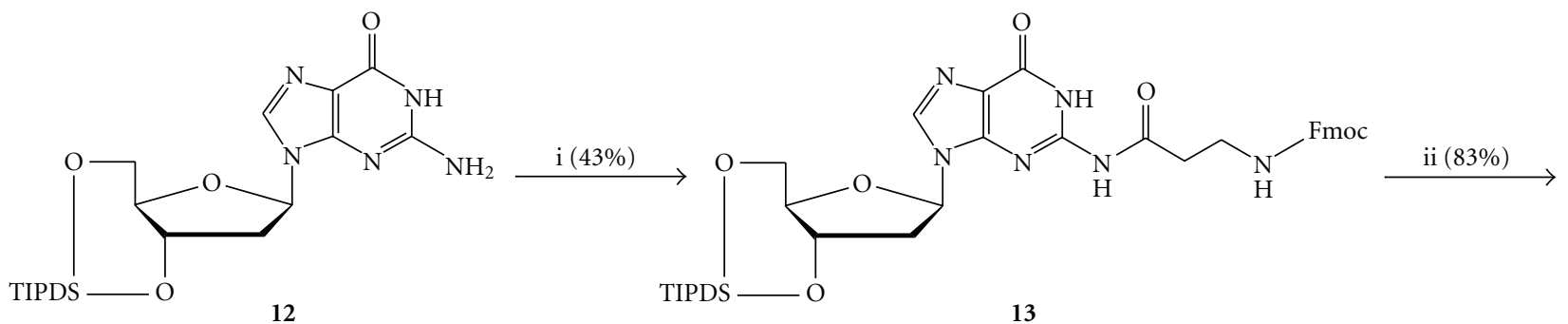<smiles></smiles>

14

Scheme 2: Reaction conditions (i) 3-(Fmoc-amino)propionyl chloride (prepared from $N$-Fmoc- $\beta$-alanine and SOCL $_{2}$ ), THF/Pyridine, rt, 72 hours. (ii) 10, EDCI, DMAP, DMF, rt, 24 hours. (iii) $0.2 \mathrm{M}$ TEAF, THF, rt, 30 minutes.

desired pyrrolepolyamide-2'-deoxyguanosine derivative 21 in $81 \%$ yield through deprotection of the Fmoc group of $\mathbf{1 9}$ and condensation of the amine product with 20 . Cleavage of the NPE protecting group using 0.5 M DBU in pyridine [28] and subsequent deacetylation using sodium methoxide in methanol generated Hybrid $\mathbf{3}$ in good yield. 
<smiles>O=C(Cl)OCC1c2ccccc2-c2ccccc21</smiles>

15 (Fmoc-Cl)

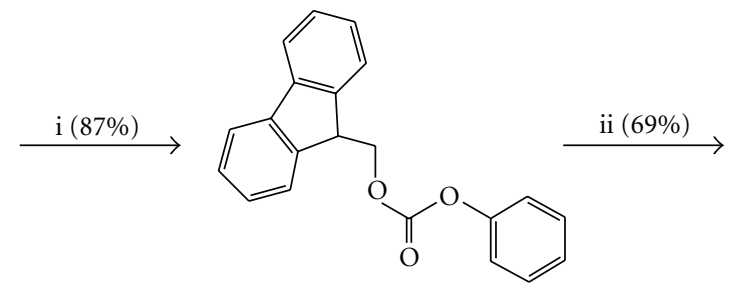

16<smiles>[NH3+]CCCNC(=O)OCC1c2ccccc2-c2ccccc21</smiles>

17<smiles>[R]Nc1cc(C(=O)Nc2cc(C(=O)Nc3cc(C(=O)NCCCNC(F)F)n(C)c3)n(C)c2)n(C)c1</smiles>

Scheme 3: Reaction conditions (i) sodium phenoxide, 1,4-dioxane, rt, 1.5 hours. (ii) 1. Propane-1,3-diamine (1.1 eq.), rt, 3.5 hours; 2. pyridinium hydrochloride. (iii) 17, DCC, HOBT, DIEA, DMF, rt, 24 hours. (v) 1. AcCL, MeOH, $0^{\circ} \mathrm{C}, 30$ minutes; 2 . HCOOH, CDI, THF, $-40^{\circ} \mathrm{C}, 1$ hour.

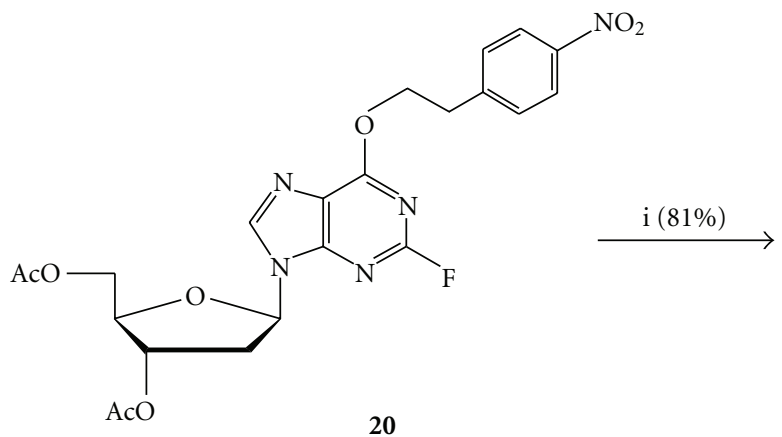

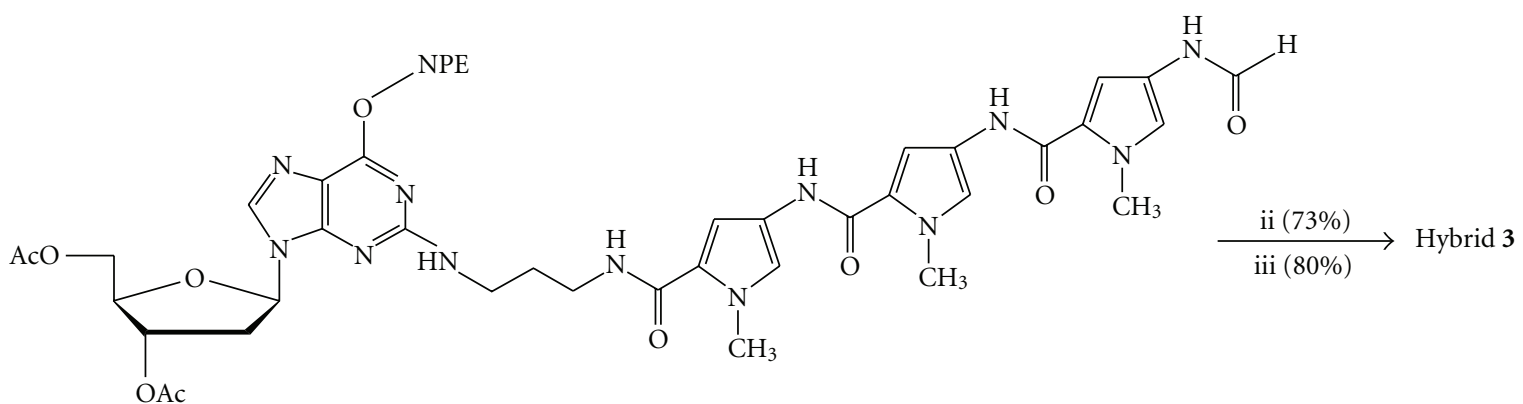

21

Scheme 4: Reaction conditions (i) 1. piperidine, DMF, rt, 20 minutes. 2. 19, $\mathrm{Et}_{3} \mathrm{~N}, \mathrm{DMF}, 60^{\circ} \mathrm{C} 12$ hours. (ii) $0.5 \mathrm{M} \mathrm{DBU}$, pyridine, rt, 12 hours. (iii) $0.05 \mathrm{M} \mathrm{NaOMe}, \mathrm{MeOH} /$ pyridine, rt, 2 hours.

For our investigations concerning the interaction of DNA duplexes with Hybrid 2 and Hybrid $\mathbf{3}$ using CD and UV melting data $\left(T_{\mathrm{m}}\right.$ value) [29], DNA 1 [d-(5'-CGCAAATTGGC$\left.3^{\prime}\right)$-d-( $\left(3^{\prime}-\right.$ GCGTTTAACCG-5' $)$, which contains the high affinity binding site of Dst, DNA 2 [d-(5' -CGCAGACTGGC$\left.3^{\prime}\right)$-d-( $3^{\prime}$-GCGTCTGACCG-5')], which included changes at A 5 to $\mathrm{G} 5$ and $\mathrm{T} 7 \mathrm{to} \overline{\mathrm{C}}$, and DNA 3 [d-(5'-GCACGACTACG$\left.3^{\prime}\right)$-d-( $3^{\prime}$-CGTGCTGATGC-5')], which represents a random 


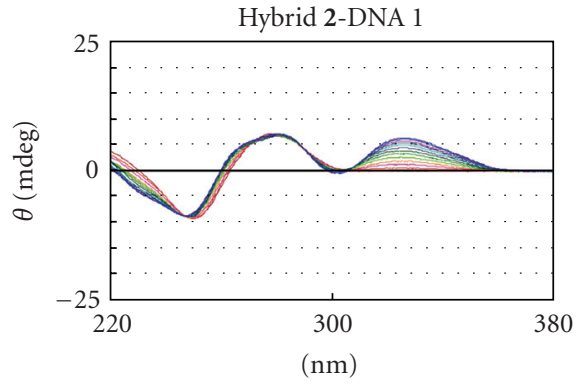

(a)

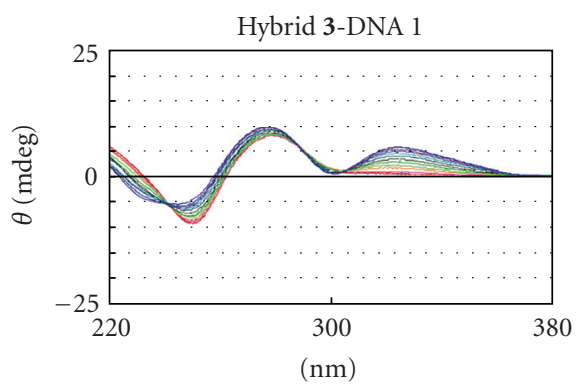

(d)

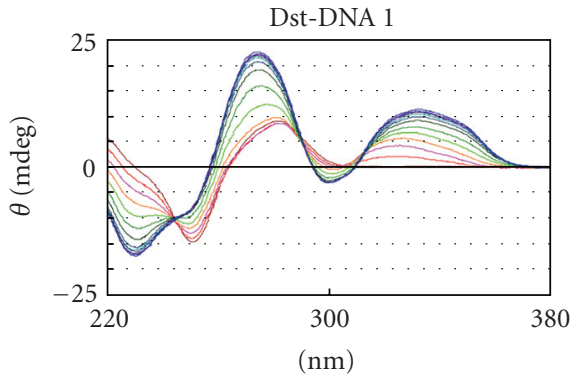

(g)

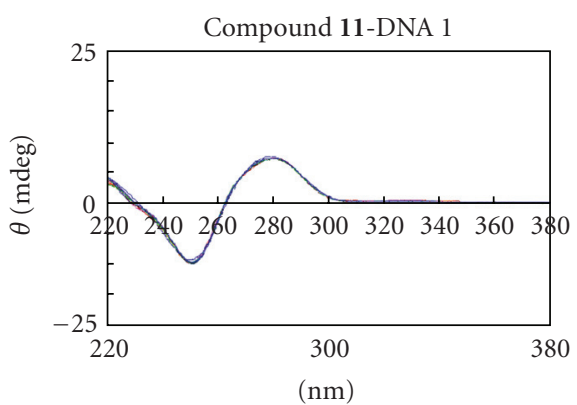

(j)

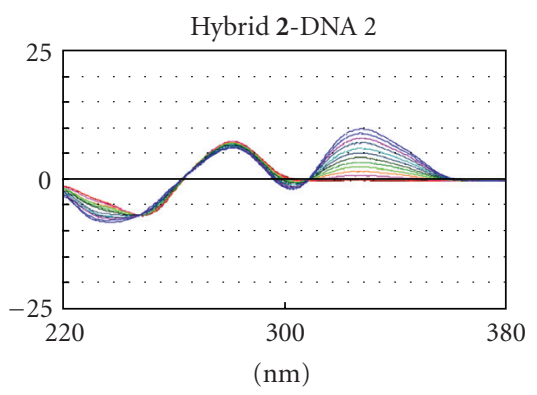

(b)

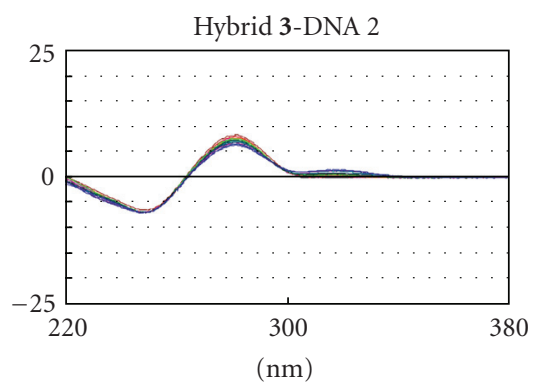

(e)

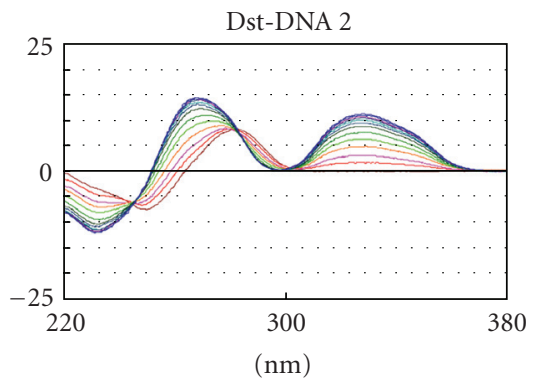

(h)

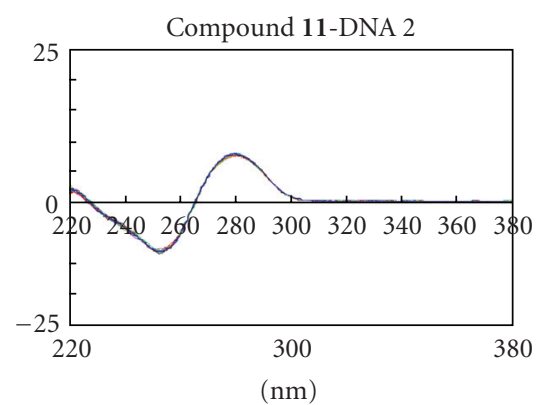

(k)

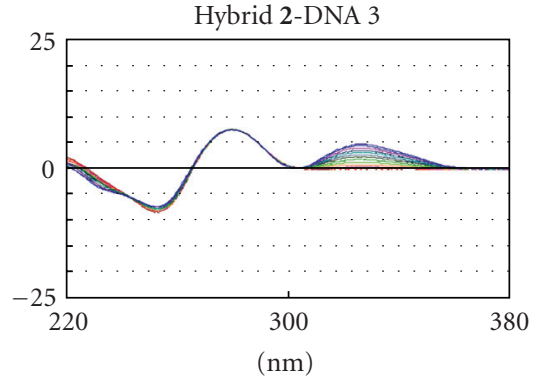

(c)

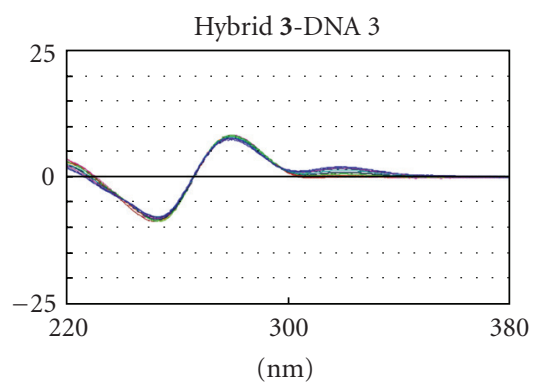

(f)

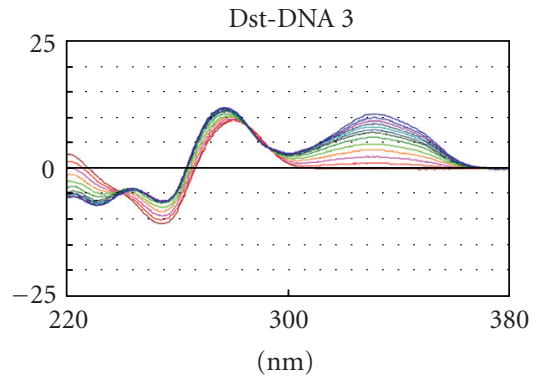

(i)

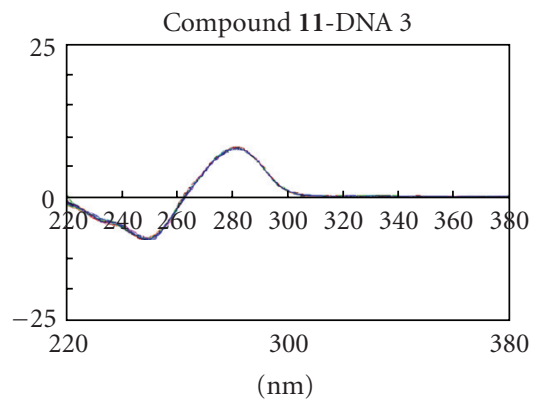

(1)

FIGURe 2: CD spectra for the DNA duplexs with ligand (Hybrid 2, Hybrid 3, Dst, or compound 11) at several [Iigand]/[duplex] ratios in buffer consisting of $10 \mathrm{mM}$ sodium phosphate, $10 \mathrm{mM} \mathrm{NaCL}$, and $0.1 \mathrm{mM} \mathrm{Na} 2 \mathrm{EDTA}$ at $\mathrm{pH} 7.0$ and $20^{\circ} \mathrm{C}$ : $3.0 \mathrm{~mL}$ of duplex solution $(5.8 \mu \mathrm{M})$ titrated ligand (Hybrid 2, Hybrid 3, and Dst: $0-14.5 \mu \mathrm{M})$ and $3.0 \mathrm{~mL}$ of duplex solution $(4 \mu \mathrm{M})$ titrated ligand (compound 11: 0-12 $\mu \mathrm{M})$. * DNA duplexs: DNA 1 = d-(5'-CGCAAATTGGC-3')-d- $\left(3^{\prime}-\right.$ GCGTTTAACCG-5'), DNA 2 = d- $\left(5^{\prime}\right.$-CGCAGACTGGC-3')-d- $\left(3^{\prime}\right.$ GCGTCTGACCG-5'), DNA 3 = d-(5'-GCACGACTACG-3')-d-(3'-CGTGCTGATGC-5' $)$, DNA 1 contains the high affinity binding site $\left(5^{\prime}\right.$ AAATT- $\left.3^{\prime}\right)$ of Dst.

sequence, were used (Figure 2 and Table 1). Moreover, experiments employing Dst and compound 11 were conducted for comparison. Typical CD spectra of the uncomplexed DNA duplexes (DNA 1-3), indicative of DNA in the B-form conformation, are shown in Figure 2 (ligand $=0 \mu \mathrm{M}$ ). The addition of Hybrid 2, Hybrid 3, or Dst resulted in marked changes in the ellipticity $\theta$, unlike the case with compound 11. In particular, an induced Cotton effect of bound ligand is indicated by the presence of an extra CD band centered at $\sim 325 \mathrm{~nm}$ for Hybrid 2 and Hybrid 3, and $\sim 333 \mathrm{~nm}$ for 
TABLE 1: $\Delta T_{m}$ values $\left({ }^{\circ} \mathrm{C}\right.$ ) for the DNA duplexs with ligand (Hybrid 1, Hybrid 2, Hybrid 3, or Dst) at several [ligand]/[duplex] ratios in buffer consisting of $10 \mathrm{mM}$ sodium phosphate, $10 \mathrm{mM} \mathrm{NaCl}$, and $0.1 \mathrm{mM} \mathrm{Na}_{2}$ EDTA at pH 7.0.

\begin{tabular}{lcccc}
\hline [ligand]/[duplex] & & $1: 1$ & $2: 1$ & $3: 1$ \\
\hline \multirow{3}{*}{ Hybrid 1 } & DNA 1 & 2.1 & 2.3 & - \\
& DNA 2 & -0.1 & 0.2 & - \\
\hline \multirow{3}{*}{ Hybrid 2 } & DNA 3 & -0.4 & 0.3 & - \\
& DNA 1 & 2.7 & 3.8 & 3.9 \\
& DNA 2 & -0.3 & -0.2 & -0.1 \\
Hybrid 3 & DNA 3 & 0.2 & 0.3 & 0.3 \\
\hline \multirow{3}{*}{ Dst } & DNA 1 & 5.6 & 5.7 & 5.8 \\
& DNA 2 & 0.1 & 0.2 & 0.5 \\
& DNA 3 & -0.3 & -0.5 & -0.2 \\
\hline & DNA 1 & 22.9 & 22.9 & 23.5 \\
& DNA 2 & 3.0 & 6.5 & 6.9 \\
& DNA 3 & 15.2 & 16.3 & 16.3 \\
\hline
\end{tabular}

${ }^{*} \Delta T_{\mathrm{m}}$ value $=\left(T_{\mathrm{m}}\right.$ value in the presence of ligand $)-\left(T_{\mathrm{m}}\right.$ value in the absence of ligand).

** DNA duplexs: DNA $1=\mathrm{d}-\left(5^{\prime}\right.$-CGCAAATTGGC-3' $)$-d- $\left(3^{\prime}\right.$ GCGTTTAACCG $-5^{\prime}$ ) $T_{\mathrm{m}}=32.2^{\circ} \mathrm{C}$.

DNA $2=\mathrm{d}-\left(5^{\prime}\right.$-CGCAGACTGGC- $\left.3^{\prime}\right)-\mathrm{d}-\left(3^{\prime}\right.$-GCGTCTGACCG- $\left.5^{\prime}\right) T_{\mathrm{m}}=$ $39.5^{\circ} \mathrm{C}$.

DNA $3=\mathrm{d}-\left(5^{\prime}-\right.$ GCACGACTACG- $\left.3^{\prime}\right)-\mathrm{d}-\left(3^{\prime}-\right.$ CGTGCTGATGC-5' $) T_{\mathrm{m}}=$ $33.9^{\circ} \mathrm{C}$.

DNA 1 contains the high affinity binding site $\left(5^{\prime}\right.$-AAATT $\left.-3^{\prime}\right)$ of Dst.

Dst. Hybrid 3 showed selective binding affinity only with DNA 1, which contains the AAATT sequence (Figure 2), and stabilization of the DNA duplex (Table 1). On the other hand, Hybrid 2, combined with a pyrrolepolyamide at the 2exocyclic amino group of the guanine base through an amide bond using a 3-aminopropionyl linker, and Dst containing the cationic amidine edge which contributes to high DNA binding affinity for ligand binding [30-33], did not show any marked differences in sequence-specific binding affinity with the tested DNA.

\section{Conclusions}

We synthesized and evaluated pyrrolepolyamide-2' deoxyguanosine hybrids (Hybrid 2 and Hybrid 3) incorporating 3-aminopropionyl or 3-aminopropyl linkers. Hybrid 3, combined with a pyrrolepolyamide at the 2exocyclic amino group of the guanine base through an $N$-alkyl bond using a 3-aminopropyl linker, showed high recognition of DNA containing the pyrrolepolyamide binding sequence. These results suggest that improvements in stability might be effected by varying the length of the linker. On the other hand, in a DNA strand with incorporated hybrid during DNA biosynthesis, the presence of a short linker, such as the 3-aminopropionyl moiety, might result in high stabilization of the dsDNA. These studies are currently underway and will be reported elsewhere. The minor groove binder pyrrolepolyamide-2'-deoxyguanosine hybrid presented in this report might potentially be of use as a sequence-specific gene therapy agent and could provide the basis for the development of a new series of antisense or antigene drugs.

\section{Acknowledgment}

This work was supported in part by a grant for private universities provided by the Promotion and Mutual Aid Corporation for Private Schools of Japan.

\section{References}

[1] P. D. Cook, "A brief history, status, and perspective of modified oligonucleotides for chemotherapeutic applications," in Current Protocols in Nucleic Acid Chemistry, S. L. Beaucage, D. E. Bergstrom, G. D. Glick, and R. A. Jones, Eds., pp. 4.1.1-4.1.17, John Wiley \& Sons, New York, NY, USA, 2000.

[2] L. Huang, J. C. Quada Jr., and J. W. Lown, "Functional models of the antitumor antibiotic bleomycin," Current Medicinal Chemistry, vol. 2, no. 1, pp. 543-560, 1995.

[3] E. E. Baird and P. B. Dervan, "Solid phase synthesis of polyamides containing imidazole and pyrrole amino acids," Journal of the American Chemical Society, vol. 118, no. 26, pp. 6141-6146, 1996.

[4] H. Sugiyama, C. Lian, M. Isomura, I. Saito, and A. H.-J. Wang, "Distamycin A modulates the sequence specificity of DNA alkylation by duocarmycin A," Proceedings of the National Academy of Sciences of the United States of America, vol. 93, no. 25, pp. 14405-14410, 1996.

[5] Y. Wang, R. Gupta, L. Huang, W. Luo, and J. W. Lown, "Design, synthesis, cytotoxic properties and preliminary DNA sequencing evaluation of CPI-N-methylpyrrole hybrids. Enhancing effect of a trans double bond linker and role of the terminal amide functionality on cytotoxic potency," AntiCancer Drug Design, vol. 11, no. 1, pp. 15-34, 1996.

[6] J. M. Gottesfeld, L. Neely, J. W. Trauger, E. E. Baird, and P. B. Dervan, "Regulation of gene expression by small molecules," Nature, vol. 387, no. 6629, pp. 202-205, 1997.

[7] J. W. Trauger, E. E. Baird, and P. B. Dervan, "Recognition of 16 base pairs in the minor groove of DNA by a pyrrole-imidazole polyamide dimer," Journal of the American Chemical Society, vol. 120, no. 14, pp. 3534-3535, 1998.

[8] Z.-F. Tao, T. Fujiwara, I. Saito, and H. Sugiyama, "Rational design of sequence-specific DNA alkylating agents based on duocarmycin A and pyrrole-imidazole hairpin polyamides," Journal of the American Chemical Society, vol. 121, no. 21, pp. 4961-4967, 1999.

[9] T. Yamori, A. Matsunaga, S. Sato, et al., "Potent antitumor activity of MS-247, a novel DNA minor groove binder, evaluated by an in vitro and in vivo human cancer cell line panel," Cancer Research, vol. 59, no. 16, pp. 4042-4049, 1999.

[10] T. Fujiwara, Z.-F. Tao, Y. Ozeki, et al., "Modulation of sequence specificity of duocarmycin-dependent DNA alkylation by pyrrole-imidazole triamides," Journal of the American Chemical Society, vol. 121, no. 33, pp. 7706-7707, 1999.

[11] D. L. Boger, B. E. Fink, and M. P. Hedrick, "Total synthesis of distamycin A and 2640 analogues: a solution-phase combinatorial approach to the discovery of new, bioactive DNA binding agents and development of a rapid, high-throughput screen for determining relative DNA binding affinity or DNA binding sequence selectivity," Journal of the American Chemical Society, vol. 122, no. 27, pp. 6382-6394, 2000. 
[12] M. A. Marques, R. M. Doss, A. R. Urbach, and P. B. Dervan, "Toward an understanding of the chemical etiology for DNA minor-groove recognition by polyamides," Helvetica Chimica Acta, vol. 85, no. 12, pp. 4485-4517, 2002.

[13] J. M. Belitsky, S. J. Leslie, P. S. Arora, T. A. Beerman, and P. B. Dervan, "Cellular uptake of N-methylpyrrole/Nmethylimidazole polyamide-dye conjugates," Bioorganic and Medicinal Chemistry, vol. 10, no. 10, pp. 3313-3318, 2002.

[14] P. G. Baraldi, R. Romagnoli, A. E. Guadix, et al., "Design, synthesis, and biological activity of hybrid compounds between uramustine and DNA minor groove binder distamycin A," Journal of Medicinal Chemistry, vol. 45, no. 17, pp. 3630-3638, 2002.

[15] B. S. Edelson, T. P. Best, B. Olenyuk, et al., "Influence of structural variation on nuclear localization of DNA-binding polyamide-fluorophore conjugates," Nucleic Acids Research, vol. 32, no. 9, pp. 2802-2818, 2004.

[16] P. G. Baraldi, M. del Carmen Nunez, M. A. Tabrizi, et al., "Design, synthesis, and biological evaluation of hybrid molecules containing $\alpha$-methylene- $\gamma$-butyrolactones and polypyrrole minor groove binders," Journal of Medicinal Chemistry, vol. 47, no. 11, pp. 2877-2886, 2004.

[17] K. Seio, M. Mizuta, T. Terada, and M. Sekine, "Use of ferrocene scaffolds as pendant groups in hairpin-type pyrrole-imidazole polyamide molecules showing sequence-selective binding to DNA duplexes," Journal of Organic Chemistry, vol. 70, no. 25, pp. 10311-10322, 2005.

[18] C. C. O'Hare, P. Uthe, H. Mackay, et al., "Sequence recognition in the minor groove of DNA by covalently linked formamido imidazole-pyrrole-imidazole polyamides: effect of $\mathrm{H}$-pin linkage and linker length on selectivity and affinity," Biochemistry, vol. 46, no. 42, pp. 11661-11670, 2007.

[19] E. Kawashima, D. Itoh, K. Kamaike, Y. Terui, and T. Oshima, "Synthesis and analysis of nucleosides bearing pyrrolepolyamide binding to DNA," Nucleosides, Nucleotides \& Nucleic Acids, vol. 22, no. 5-8, pp. 1309-1311, 2003.

[20] Y. Ohba, K. Kamaike, Y. Terui, T. Oshima, and E. Kawashima, "Design, synthesis and analysis of antiviral nucleosides bearing pyrrolepolyamide binding to nucleic acid (II): $\mathrm{N}^{2}$ pyrrolepolyamidopropylguanosine," Nucleic Acids Research Supplement, no. 3, pp. 29-30, 2003.

[21] M. Mrksich, M. E. Parks, and P. B. Dervan, "Hairpin peptide motif. A new class of oligopeptides for sequence-specific recognition in the minor groove of double-helical DNA," Journal of the American Chemical Society, vol. 116, no. 18, pp. 7983-7988, 1994.

[22] W. T. Markiewicz, "Tetraisopropyldisiloxane-1,3-diyl, a group for simultaneous protection of $3^{\prime}$ - and $5^{\prime}$-hydroxy functions of nucleosides," Journal of Chemical Research (S), pp. 24-25, 1979.

[23] W. T. Markiewicz, "Tetraisopropyldisiloxane-1,3-diyl, a group for simultaneous protection of $3^{\prime}$ - and 5' -hydroxy functions of nucleosides," Journal of Chemical Research (M), pp. 181-197, 1979.

[24] H. Lee, M. Hinz, J. J. Stezowski, and R. G. Harvey, "Syntheses of polycyclic aromatic hydrocarbon-nucleoside and oligonucleotide adducts specifically alkylated on the amino functions of deoxyguanosine and deoxyadenosine," Tetrahedron Letters, vol. 31, no. 47, pp. 6773-6776, 1990.

[25] A. Adib, P. F. Potier, S. Doronina, I. Huc, and J.-P. Behr, "A high-yield synthesis of deoxy-2-fluoroinosine and its incorporation into oligonucleotides," Tetrahedron Letters, vol. 38, no. 17, pp. 2989-2992, 1997.
[26] K. Kamaike, K. Kinoshita, K. Niwa, K. Hirose, K. Suzuki, and Y. Ishido, "Efficient methods for the synthesis of [2$\left.{ }^{15} \mathrm{~N}\right]$ guanosine and 2 -deoxy $\left[2-{ }^{15} \mathrm{~N}\right]$ guanosine derivatives," Nucleosides, Nucleotides \& Nucleic Acids, vol. 20, no. 1-2, pp. 59-75, 2001.

[27] M. Pittelkov, R. Lewinsky, and J. B. Christensen, "Selective synthesis of carbamate protected polyamines using alkyl phenyl carbonates," Synthesis, pp. 2195-2202, 2002.

[28] B. L. Gaffney and R. A. Jones, "A new strategy for the protection of deoxyguanosine during oligonucleotide synthesis," Tetrahedron Letters, vol. 23, no. 22, pp. 2257-2260, 1982.

[29] D. Rentzeperis, L. A. Marky, T. J. Dwyer, B. H. Geierstanger, J. G. Pelton, and D. E. Wemmer, "Interaction of minor groove ligands to an AAATT/AATTT site: correlation of thermodynamic characterization and solution structure," Biochemistry, vol. 34, no. 9, pp. 2937-2945, 1995.

[30] J. G. Pelton and D. E. Wemmer, "Binding modes of distamycin A with d(CGCAAATTTGCG)2 determined by twodimensional NMR," Journal of the American Chemical Society, vol. 112, no. 4, pp. 1393-1399, 1990.

[31] J. G. Pelton and D. E. Wemmer, "Structural modeling of the distamycin A-d(CGCGAATTCGCG)2 Complex using 2D NMR and molecular mechanics," Biochemistry, vol. 27, no. 21, pp. 8088-8096, 1988.

[32] M. Coll, C. A. Frederick, A. H. Wang, and A. Rich, "A bifurcated hydrogen-bonded conformation in the $\mathrm{d}(\mathrm{A} . \mathrm{T})$ base pairs of the DNA dodecamer d(CGCAAATTTGCG) and its complex with distamycin," Proceedings of the National Academy of Sciences of the United States of America, vol. 84, no. 23, pp. 8385-8389, 1987.

[33] M. L. Kopka, C. Yoon, D. Goodsell, P. Pjura, and R. E. Dickerson, "The molecular origin of DNA-drug specificity in netropsin and distamycin," Proceedings of the National Academy of Sciences of the United States of America, vol. 82, no. 5, pp. 1376-1380, 1985. 

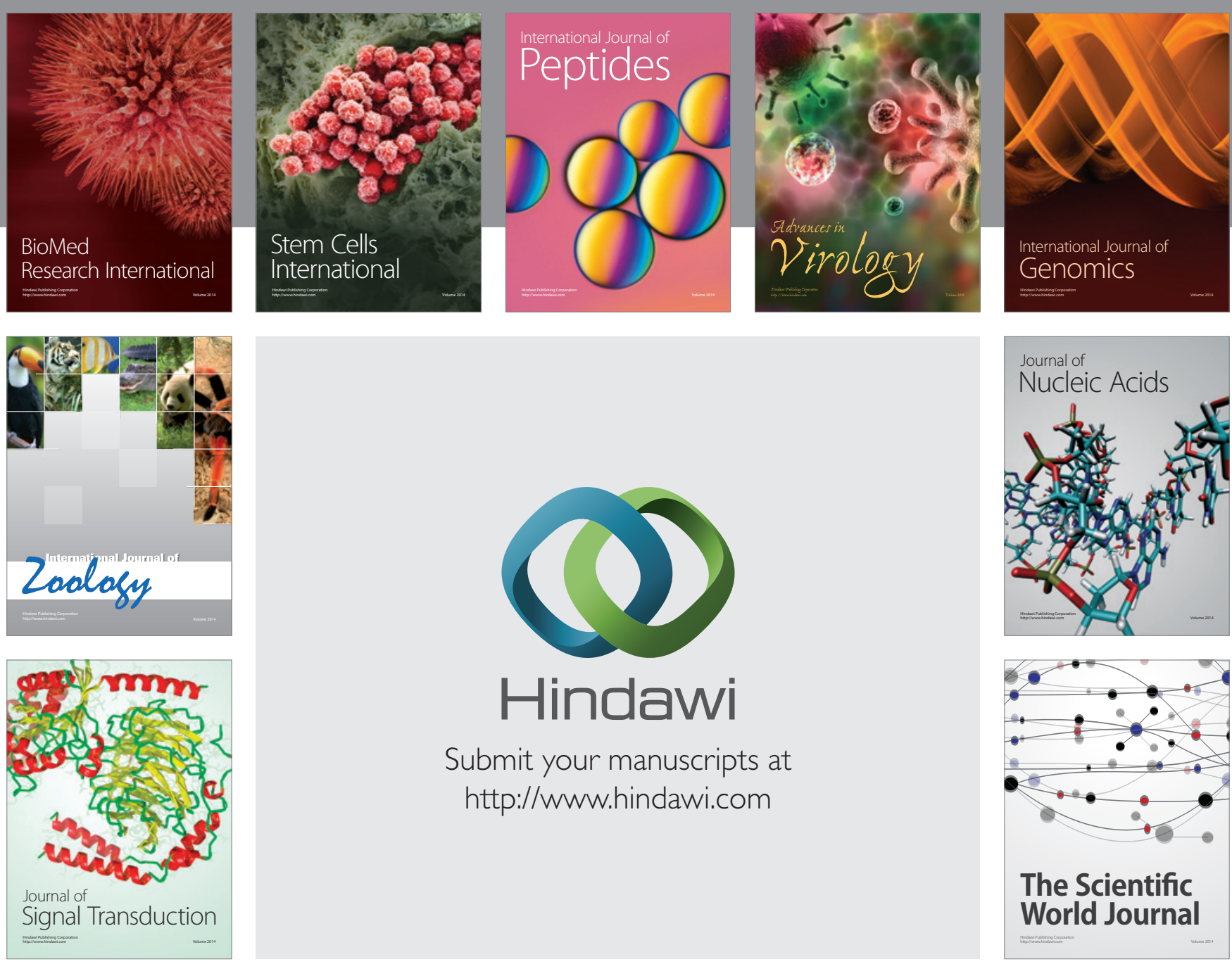

Submit your manuscripts at

http://www.hindawi.com
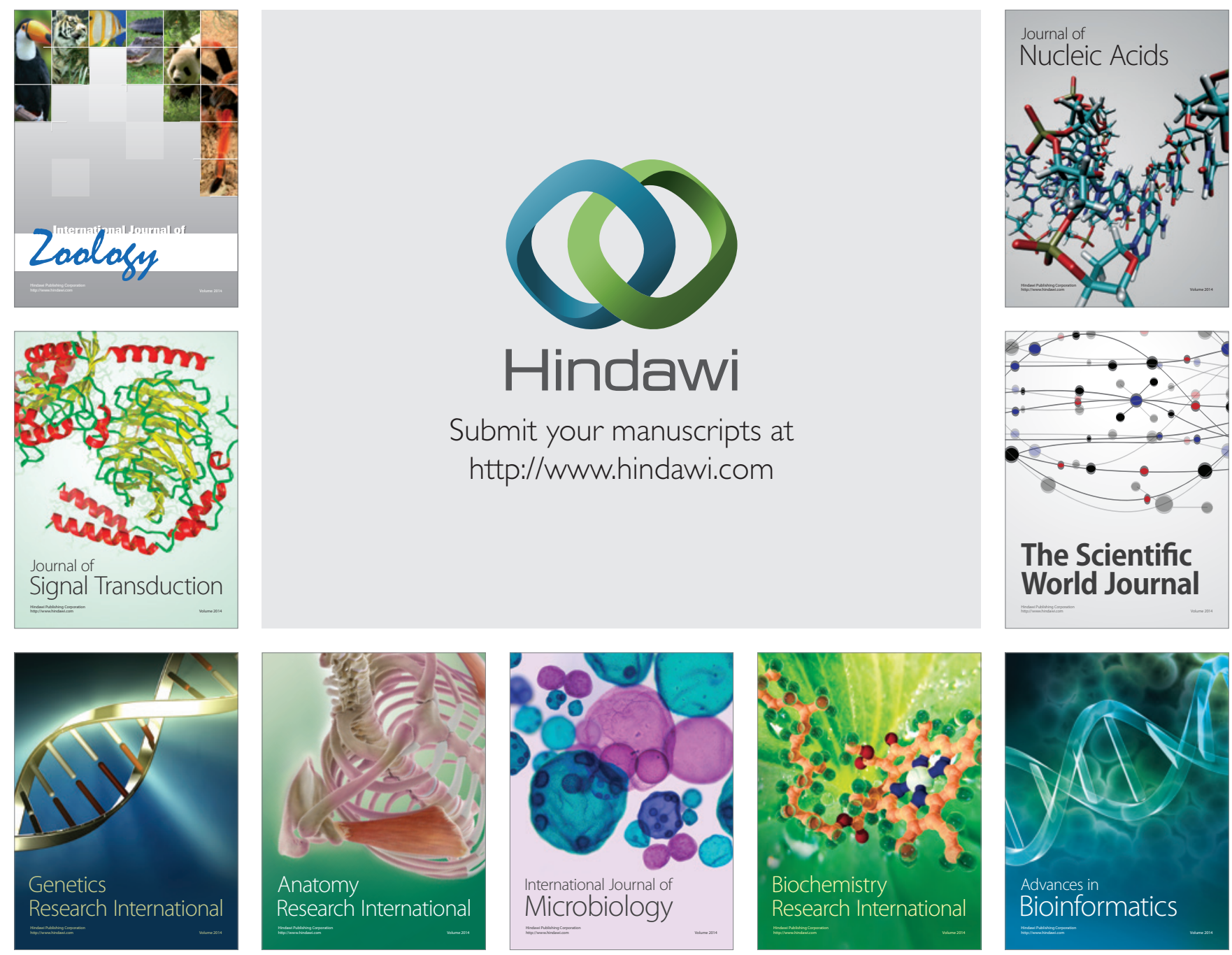

The Scientific World Journal
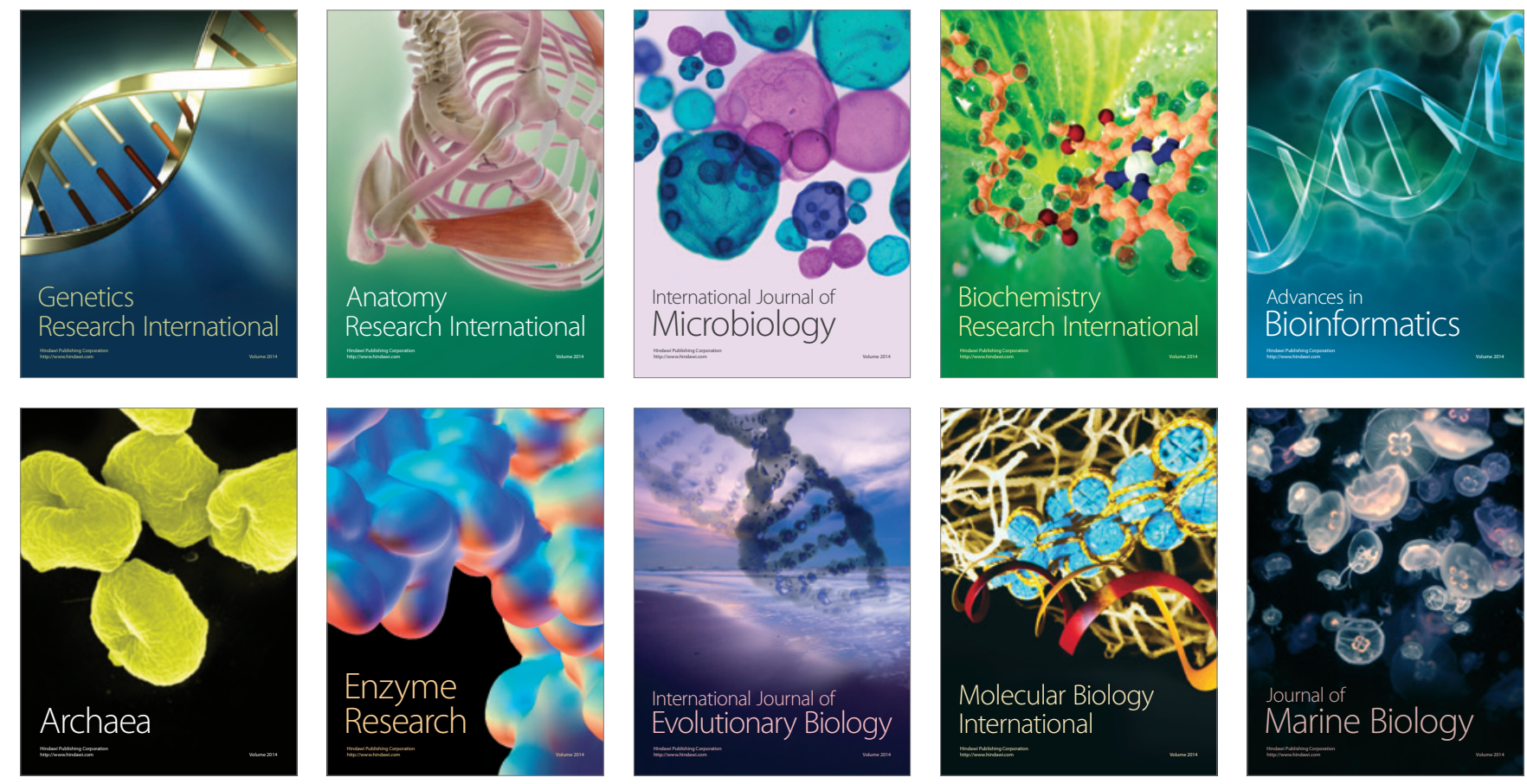